\title{
Analysis of the Quiescent Low\#Mass X\#Ray Binary Population in Galactic Globular Clusters
}

\section{Citation}

Heinke, C. O., J. E. Grindlay, P. M. Lugger, H. N. Cohn, P. D. Edmonds, D. A. Lloyd, and A. M. Cool. 2003. "Analysis of the Quiescent Low\#Mass X\#Ray Binary Population in Galactic Globular Clusters." The Astrophysical Journal 598 (1): 501-15. https://doi.org/10.1086/378885.

\section{Permanent link}

http://nrs.harvard.edu/urn-3:HUL.InstRepos:41399894

\section{Terms of Use}

This article was downloaded from Harvard University's DASH repository, and is made available under the terms and conditions applicable to Other Posted Material, as set forth at http:// nrs.harvard.edu/urn-3:HUL.InstRepos:dash.current.terms-of-use\#LAA

\section{Share Your Story}

The Harvard community has made this article openly available.

Please share how this access benefits you. Submit a story.

Accessibility 


\title{
ANALYSIS OF THE QUIESCENT LOW-MASS X-RAY BINARY POPULATION IN GALACTIC GLOBULAR CLUSTERS
}

\author{
C. O. Heinke, ${ }^{1}$ J. E. Grindlay, ${ }^{1}$ P. M. Lugger ${ }^{2}$ H. N. Cohn, ${ }^{2}$ P. D. Edmonds, ${ }^{1}$ \\ D. A. Lloyd, ${ }^{1}$ AND A. M. CoOL ${ }^{3}$ \\ Received 2003 May 22; accepted 2003 August 4
}

\begin{abstract}
Quiescent low-mass X-ray binaries (qLMXBs) containing neutron stars have been identified in several globular clusters using Chandra or $X M M$ X-ray observations, via their distinctive soft thermal spectra. We report a complete census of the qLMXB population in these clusters, identifying three additional probable qLMXBs in NGC 6440. We conduct several analyses of the qLMXB population and compare it with the harder, primarily cataclysmic variable (CV), population of low-luminosity X-ray sources with $10^{31} \mathrm{ergs} \mathrm{s}^{-1}<L_{\mathrm{X}}<10^{32.5} \mathrm{ergs} \mathrm{s}^{-1}$. The radial distribution of our qLMXB sample suggests an average system mass of $1.5_{-0.2}^{+0.3} M_{\odot}$, consistent with a neutron star and low-mass companion. Spectral analysis reveals that no globular cluster qLMXBs, other than the transient in NGC 6440, require an additional hard powerlaw component, as often observed in field qLMXBs. We identify an empirical lower luminosity limit of $\sim 10^{32}$ ergs $\mathrm{s}^{-1}$ among globular cluster qLMXBs. The bolometric luminosity range of qLMXBs implies (in the deep crustal heating model of Brown and collaborators) low time-averaged mass-transfer rates, below the disk stability criterion. The X-ray luminosity functions of the CV populations alone in NGC 6397 and 47 Tuc are shown to differ. The distribution of qLMXBs among globular clusters is consistent with their dynamical formation by either tidal capture or exchange encounters, allowing us to estimate that 7 times more qLMXBs than bright LMXBs reside in globular clusters. The distribution of harder sources (primarily CVs) has a weaker dependence on density than that of the qLMXBs. Finally, we discuss possible effects of core collapse and globular cluster destruction on X-ray source populations.
\end{abstract}

Subject headings: globular clusters: general — globular clusters: individual (NGC 6440) novae, cataclysmic variables - stars: neutron - stellar dynamics - X-rays: binaries

\section{INTRODUCTION}

Globular clusters have proved to be an excellent place to study accreting binary systems, because of their known distances, ages, and reddening, which allow system parameters and histories to be better understood than in the field. It has long been suspected that globular clusters may also provide unique channels for the formation of accreting binaries, starting with the discovery that bright (observed at $L_{\mathrm{X}}>10^{36} \mathrm{ergs} \mathrm{s}^{-1}$ ) low-mass X-ray binaries (LMXBs) are $\sim 100$ times more common (per unit mass) in globular clusters than in the field (Clark 1975; Katz 1975). It is now thought that X-ray binary systems in dense globular clusters are created principally through exchange interactions between primordial binaries and other stars (see, e.g., Hut, Murphy, \& Verbunt 1991). Therefore, studying accreting binary systems in globular clusters can give us insight into the characteristics of accreting binary systems and populations and also into dynamical effects inside globular clusters.

The giant leap forward in sensitivity and angular resolution offered by the Chandra X-Ray Observatory, especially when combined with the unparalleled resolution of the

\footnotetext{
${ }^{1}$ Harvard-Smithsonian Center for Astrophysics, 60 Garden Street, Cambridge, MA 02138; cheinke@cfa.harvard.edu, josh@cfa.harvard.edu, pedmonds@cfa.harvard.edu,dlloyd@cfa.harvard.edu.

${ }^{2}$ Department of Astronomy, Indiana University, Swain West 319, Bloomington, IN 47405; lugger@indiana.edu,cohn@indiana.edu.

${ }^{3}$ San Francisco State University, 1600 Holloway Avenue, San Francisco, CA 94132; cool@sfsu.edu.
}

Hubble Space Telescope (HST), has revolutionized our understanding of globular clusters. Prior to Chandra, there were 12 bright cluster LMXBs (now 13, all thought to have neutron stars [NSs] as primaries) and 57 faint X-ray sources known in the Galactic globular cluster system (Verbunt 2001). A few of the latter had been identified with cataclysmic variables (CVs) (Hertz \& Grindlay 1983; Cool et al. 1995; Grindlay et al. 1995), and some were thought to be LMXBs in quiescence (qLMXBs; Verbunt, Elson, \& van Paradijs 1984), but their properties were poorly understood because of the poor spatial and/or spectral resolution of previous X-ray observatories. Chandra has identified more than $100 \mathrm{X}$-ray sources in the globular cluster 47 Tuc alone (Grindlay et al. 2001a, hereafter GHE01a). Dozens of sources have been discovered in the globular clusters $\omega$ Cen (Rutledge et al. 2002a; Cool, Haggard, \& Carlin 2002), NGC 6397 (Grindlay et al. 2001b, hereafter GHE01b), NGC 6752 (Pooley et al. 2002a), NGC 6440 (Pooley et al. 2002b; in 't Zand et al. 2001), M28 (Becker et al. 2003), Terzan 5 (Heinke et al. 2003a), and M80 (Heinke et al. 2003b). These plus preliminary results from several additional clusters create a sizable data set for comparison of $\mathrm{X}$-ray source populations with cluster properties (Pooley et al. 2003), ${ }^{4}$ especially when combined with initial $X M M$ results on the low-density clusters M22, $\omega$ Cen, and M13 (Webb, Gendre, \& Barret 2002; Gendre et al. 2003a, 2003b). $H S T$ identifications have allowed the classification of many

\footnotetext{
${ }^{4}$ See D. Pooley et al. 2003, at http://online.kitp.ucsb.edu/online/clusters_c03.
} 
as CVs or active main-sequence or subgiant binaries (ABs) in 47 Tuc (Edmonds et al. 2003a, 2003b), NGC 6397 (GHE01b), NGC 6752 (Pooley et al. 2002a), and $\omega$ Cen (Cool, Haggard, \& Carlin 2002), and one as a qLMXB in 47 Tuc (Edmonds et al. 2002a). Radio-derived positions or orbital periods for millisecond pulsars (MSPs) have allowed the identification of faint X-ray sources as MSPs in 47 Tuc (Edmonds et al. 2001; Grindlay et al. 2002; Edmonds et al. 2002a), NGC 6397 (GHE01b), and NGC 6752 (D'Amico et al. 2002). However, radio and $H S T$ coverage of globular clusters is generally quite incomplete, and faint $\mathrm{X}$-ray sources in most globular clusters do not have clear identifications from other wave bands. Even with deep radio and optical data, MSPs may be confused with CVs (see Edmonds et al. 2002a) or with ABs (Ferraro et al. 2001; but cf. Edmonds et al. 2003b). However, qLMXBs show clear differences from all other types of globular cluster X-ray sources in their colors and luminosities $\left(L_{X} \sim 10^{32}-10^{34}\right.$ ergs $\mathrm{s}^{-1}$, with soft X-ray colors) and detailed spectra (a thermal component with an implied radius of a few kilometers, sometimes accompanied by a nonthermal harder component). Their X-ray emission is thought to be due principally to thermal emission from the NS surface (Brown, Bildsten, \& Rutledge 1998; Campana et al. 1998), thus making their X-ray signatures more homogeneous than those of other source types. These differences make it practical to identify a homogeneous sample of qLMXBs in different globular clusters without the need for deep HST or radio data sets, using $\mathrm{X}$-ray spectra, colors, and luminosities alone, as for instance in 47 Tuc (GHE01a), $\omega$ Cen (Rutledge et al. 2002a), NGC 6397 (GHE01b), NGC 6440 (Pooley et al. 2002b), and M28 (Becker et al. 2003).

We perform several analyses of the qLMXB population that has been uncovered in numerous globular clusters. The qLMXB population in globular clusters offers hope for understanding many questions related to NSs, accretion flows, and cluster dynamics. Among these questions are the following: What is the source of the X-ray luminosity observed from qLMXBs, deep crustal heating (Brown et al. 1998) or continued low-level accretion? What is the nature and origin of the nonthermal hard component observed in many qLMXB spectra? How long are qLMXBs in quiescence between outbursts? Are qLMXBs part of the progenitor population of MSPs? Can we constrain the radius and/ or mass of NSs in qLMXBs through observation of the thermal component of their spectra? What are the parameters of globular clusters that influence the formation rate of qLMXBs? Can their numbers be fully explained through two-body or single-binary encounter rates? Are other globular cluster X-ray sources (such as CVs) formed in the same way? We gather evidence to begin answering these questions in this paper.

In $\S 2$ we identify a sample of confirmed and potential qLMXBs containing NSs in several globular clusters and analyze their X-ray spectra. In $\S 3.1$ we study the qLMXB radial distributions in King-model clusters. In $\S 3.2$ we analyze the qLMXB (and harder source) luminosity functions, while in $\S 3.3$ we analyze the dependencies of qLMXB and harder source numbers on cluster structural parameters. In $\S 4$ we discuss the meaning of qLMXB radial distributions and spectra ( $\S 4.1$ ), qLMXB luminosities ( $\S 4.2$ ), qLMXB versus $C V$ distributions among clusters ( $\S 4.3$ ), and additional dynamical processes ( $\S 4.4)$. Finally, we summarize in $\S 5$.

\section{QUIESCENT LMXBs}

Identifying the nature of X-ray sources is often difficult, requiring deep multiwavelength data. The unique X-ray spectral signature of a qLMXB, however, offers the possibility of identifying qLMXBs without multiwavelength follow-up (important since they can be extremely optically faint; see Edmonds et al. 2002b; GHE01b). In the field, qLMXBs containing NSs have been identified after bright transient outbursts, often exhibiting type I X-ray bursts confirming their NS nature. (See Campana et al. 1998 for a review.) Their quiescent spectra show a thermal component roughly consistent with a $10 \mathrm{~km}$ NS (when hydrogen atmospheres are taken into account, e.g., Brown et al. 1998). In addition, a harder component parameterized as a power law of photon index 1-2 is often seen, comprising up to $40 \%$ of the 0.5-10 keV emission (Campana et al. 1998; Rutledge et al. $2002 \mathrm{~b}$ and references therin). Their minimum X-ray $(0.5-2.5 \mathrm{keV})$ luminosity appears to range between $5 \times 10^{31}$ and a few $10^{33}$ ergs $\mathrm{s}^{-1}$, although distances and thus luminosities are uncertain. Comparison with field systems has thus allowed numerous qLMXBs to be identified in $\mathrm{X}$-ray studies of globular clusters (see below).

The large sample of qLMXBs now known in several globular clusters allows significant comparative study. The known distances and reddening to globular clusters allows accurate luminosities to be derived, which is generally impossible in the field. We can fit the spectra to look for a hard power-law component, as seen in some field qLMXBs, and to check for consistency with a $10 \mathrm{~km}, 1.4 M_{\odot} \mathrm{NS}$ explanation. Calibration changes since the publication of some early papers makes revisiting the spectral analysis on several clusters desirable, while a common standard for identifying qLMXBs would also be useful. For all analysis in this paper, we use photoelectric absorption X-ray cross sections of Balucinska-Church \& McCammon (1992) in the XSPEC phabs model (Arnaud 1996).

For this paper we choose to identify a "canonical" qLMXB signature, spectral consistency with a nonmagnetic hydrogen atmosphere of implied radius $\sim 10 \mathrm{~km}$, with a small ( $<40 \%$ of $0.5-10 \mathrm{keV}$ flux) contribution from a power-law component allowed. This is chosen to match qLMXB systems studied in the field (Cen X-4 [Rutledge et al. 2001], Aql X-1 [Rutledge et al. 2002b], and 4U 2129+47 [Nowak, Heinze, \& Begelman 2002]) and gives a spectrum much softer than that from known CVs. A notable exception to this signature is the millisecond X-ray pulsar SAX J1808.4-3658 (SAX J1808), which has shown an extremely faint $\left[L_{X}(0.5-10 \mathrm{keV})=5 \times 10^{31} \mathrm{ergs} \mathrm{s}^{-1}\right]$ quiescent spectrum in a recent $X M M$ observation dominated by a hard (photon index $\sim 1.5$ ) power-law component (Campana et al. 2002). We would not be able to distinguish such an object from CVs, which have similar spectra, in globular clusters. Therefore we concentrate in this paper on studying a sample of objects that we can be fairly certain are qLMXBs.

As a comparison sample, we discuss spectrally harder objects of similar X-ray luminosity. On the basis of optical analysis in several clusters (GHE01a, GHE01b; Pooley et al. 2002a; Edmonds et al. 2003a), these are thought to be mostly CVs, at least above $10^{31} \mathrm{ergs} \mathrm{s}^{-1}$ (below which active binaries and MSPs become numerous). One hard X-ray source at the upper end of this luminosity range has been identified as a bright MSP in the globular cluster M28 
(Becker et al. 2003), while a moderately hard source in 47 Tuc with an unusual spectrum and strong variability has been suggested as a qLMXB (X10) (GHE01a; Edmonds et al. 2003a). Transient black holes in quiescence have X-ray spectra and luminosities indistinguishable from those of $\mathrm{CVs}$, although none have yet been positively identified in a globular cluster.

CVs, composed of a (usually low-mass main-sequence) secondary, a white dwarf, and an accretion disk, display several optical signatures. In $U$ versus $U-V$ colormagnitude diagrams (CMDs) they appear bluer than the main sequence, usually lying between the main sequence and the white dwarf cooling sequence. A strong contributor to this blue color, the accretion disk, will also generate $\mathrm{H} \alpha$ emission and cause short-timescale nonperiodic variability (flickering) and sometimes large-amplitude outbursts. In $V$ versus $V-I C M D s, C V s$ generally appear redder because of the increased contribution of the secondary light, and in globular clusters they have often been observed to fall on or near the main sequence (Edmonds et al 2003a). The secondary, filling its Roche lobe, will often show ellipsoidal variations, and this periodic, low-amplitude signal is detectable if the noise from flickering is not too large. The X-ray to optical flux ratio for CVs should be smaller than for qLMXBs, and a $\mathrm{CV}$ at a given $\mathrm{X}$-ray luminosity will display much bluer colors than a qLMXB at the same luminosity, since it must be accreting at a much higher rate. On the basis of these characteristics, X-ray sources can be identified as CVs with high confidence.

Detailed searches for variability and/or $\mathrm{H} \alpha$ excess among blue counterparts to Chandra X-ray sources have been published for three clusters, NGC 6397, NGC 6752, and 47 Tuc. Among the population of hard X-ray sources with $L_{\mathrm{X}}(0.5-2.5)>10^{31} \mathrm{ergs} \mathrm{s}^{-1}$, six of eight sources have been optically identified as CVs in NGC 6397 (GHE01b). The two remaining objects are an active binary system and a probable $\mathrm{CV}$ (based on an $N_{\mathrm{H}}$ column above the cluster value). Similarly, six CVs have been optically identified in NGC 6752 (Pooley et al. 2002a) among eight of these sources, with the remaining two objects being one probable BY Dra system and one unidentified source. (See $\S 2.5$ for discussion of the soft source CX8 in NGC 6752.) Finally, 12 CVs have been identified among 18 of these sources in 47 Tuc (GHE01a; Edmonds et al. 2003a, 2003b). The other six include three active binary systems and three sources with marginal optical counterparts that are suggested as $\mathrm{CVs}$. Thus the fraction of non-CVs among this population seems to be less than $\sim 20 \%$ in three very different clusters.

Quiescent LMXBs have been spectrally identified in 47 Tuc (two; GHE01a), $\omega$ Cen (one; Rutledge et al. 2002a), NGC 6397 (one; GHE01b), NGC 6440 (four or five; Pooley et al. 2002b, in 't Zand et al. 2001), M28 (one; Becker et al. 2003), Terzan 5 (four plus the transient LMXB; Heinke et al. 2003a), M13 (one; Gendre et al. 2003b), M30 (one; P. M. Lugger et al. 2003, in preparation), M80 (two; Heinke et al. 2003b), and NGC 6266 (five; D. Pooley et al. 2003, in preparation). Spectral analyses have been performed for most of these. However, in several cases the work was performed before the low-energy Chandra quantum efficiency degradation was known and calibrated, so we repeat the analysis. We also attempt a census of all qLMXBs in clusters studied with Chandra or $X M M$. We summarize some important quantities and results from Chandra or $X M M$ studies of several clusters in Table 1 (with clusters listed in order of decreasing close encounter rates; see $\S \S 3.3$ and 4.3). Physical quantities are from the catalog of Harris (1996, updated in 2003), ${ }^{5}$ with a few additional updates, principally core radii and distances (referenced). We compute $N_{\mathrm{H}}$ values from $E(B-V)$ using the relation between $A_{V}$ and $N_{\mathrm{H}}$ of Predehl \& Schmitt (1995), using $R_{V}=3.1$ (Cardelli, Clayton, \& Mathis 1989) to derive $N_{\mathrm{H}}=5.5 \times$ $10^{21} E(B-V)$. After this manuscript was submitted, new estimates of the distance and reddening to three of these globular clusters were made available (Gratton et al. 2003). Using these revised values would not substantially impact our results, with one possible exception discussed in $\S 4.2$.

\subsection{Tuc and $M 30$}

These clusters have been recently analyzed (Heinke et al. 2003c; P. M. Lugger et al. 2003, in preparation) with the same nonmagnetic hydrogen-atmosphere model (Lloyd 2003) used in this work. We include the relevant results from their analyses of these qLMXBs in Table 2 . We correct the luminosities and radii for the fraction of the $1 \mathrm{keV}$ Chandra point-spread function included in the extraction circles, using the CIAO tool mkpsf. The luminosity corrections amount to factors of 1.05 and 1.10 for 47 Tuc and M30, respectively; this small correction was not applied to the 47 Tuc results in Heinke et al. (2003c). The possibility that X10 in 47 Tuc may be a qLMXB was suggested by Edmonds et al. (2003b) on the basis of its high X-ray to optical flux ratio. However, this object is dramatically variable in the X-ray (GHE01a), while the X-ray and optical flux measurements were not simultaneous (as pointed out by Edmonds et al. 2003b). Its X-ray spectrum can be described as a power law of photon index 3 , with no apparent contribution from a thermal hydrogen atmosphere component (GHE01a). We also note that the optical counterpart V3, while on the main sequence in a $V, V-I C M D$, falls near the white dwarf cooling tracks 2.8 mag blueward of the main sequence in a $U$, $U-V$ CMD (Edmonds et al. 2003a). This indicates a very strong contribution of $U$ light from the disk, similar to several other CVs apparent in the 47 Tuc CMDs of Edmonds et al. (2003a). This can be compared with the known qLMXB X5, which falls roughly 0.9 mag blueward of the main sequence in a F300W, F300W-F555W CMD (Edmonds et al. 2003a). The difference in $U$ contributions suggests that significantly more mass transfer is occurring in X10 than in X5. X5's high inclination could reduce its disk component, but the eclipsing CVs W8 and W15 also appear very blue (Edmonds et al. 2003a). Further investigation of the X-ray and optical properties of this object in the coordinated simultaneous Chandra/HST observations of 47 Tuc in late 2002 is underway and should resolve the question of X10's nature. For the purposes of this paper, we simply note that X10 does not fit our "canonical" qLMXB definition above, and thus we exclude it from our qLMXB analysis.

\section{2. $M 28$ and $M 13$}

Quiescent LMXBs in these clusters have been recently analyzed using the Zavlin, Pavlov, \& Shibanov (1996) nonmagnetic hydrogen atmosphere $n s a$ model (Becker et al. 2003; Gendre et al. 2003b). This model gives very similar results to the Lloyd (2003) model. Since the data are not yet public, we include the results of their analyses in our table.

\footnotetext{
${ }^{5}$ Available at http://physun.physics.mcmaster.ca/Globular.html.
} 
TABLE 1

Recent Chandra or XMM Globular Cluster Surveys

\begin{tabular}{|c|c|c|c|c|c|c|c|c|c|}
\hline Cluster & $\begin{array}{c}N_{\mathrm{H}} \\
\left(10^{22} \mathrm{~cm}^{-2}\right)\end{array}$ & $\begin{array}{c}D \\
\text { (kpc) }\end{array}$ & $\begin{array}{l}\log \left(\rho_{0}\right)^{\mathrm{a}} \\
\left(L_{\odot} \mathrm{pc}^{-3}\right)\end{array}$ & $\begin{array}{c}r_{c} \\
\text { (arcmin) }\end{array}$ & $\begin{array}{c}\Gamma \\
\text { (Galactic } \\
\text { percentage) }\end{array}$ & NUMber OF NSs & \multicolumn{2}{|c|}{ Number of Hard SOURCes ${ }^{b}$} & REFERENCE \\
\hline NGC $6440 \ldots \ldots .$. & 0.59 & 8.5 & 5.28 & 0.13 & 6.4 & 8 & 5 & $>16$ & 1,2 \\
\hline Ter 5 & 1.20 & 8.7 & 5.23 & 0.13 & 5.9 & $>5$ & $>4$ & $?$ & 3,4 \\
\hline NGC $6266 \ldots \ldots . .$. & 0.26 & 6.9 & $5.14 \mathrm{c} ?$ & 0.18 & 5.2 & 5 & 5 & $26^{\mathrm{b}}$ & 5,6 \\
\hline 47 Tuc ............... & 0.030 & 4.5 & 4.82 & 0.40 & 3.6 & 2 & 3 & $22^{\mathrm{b}}$ & 7,8 \\
\hline M80 .................. & 0.094 & 10.3 & 4.87 & 0.11 & 1.7 & 2 & 3 & 14 & 9 \\
\hline$\omega$ Cen & 0.09 & 5.3 & 3.03 & 3.15 & 0.64 & 1 & 2 & $\sim 9^{\mathrm{b}}$ & $12,13,14,15$ \\
\hline M30 …............... & 0.017 & 9.8 & $5.04 \mathrm{c}$ & 0.06 & 0.58 & 1 & 0 & 3 & 16 \\
\hline NGC 5904....... & 0.017 & 7.5 & 3.91 & 0.42 & 0.48 & 0 & 0 & $4^{b}$ & 5,6 \\
\hline NGC $6397 . . . . .$. & 0.10 & 2.7 & $5.41 \mathrm{c}$ & 0.08 & 0.40 & 1 & 3 & 8 & $17,18,19$ \\
\hline M22 ….................. & 0.22 & 3.2 & 3.64 & 1.42 & 0.39 & 0 & 0 & 3 & 20,21 \\
\hline M13 ................. & 0.01 & 7.7 & 3.33 & 0.78 & 0.23 & 1 & 2 & $?$ & 21,22 \\
\hline
\end{tabular}

Note.-Distances, reddening, core radius and cluster central density taken from the most recent X-ray analysis work or from the Harris 1996 catalog (updated 2003 February) with a few updates. The $N_{\mathrm{H}}$ values are calculated from $E(B-V)$ values using the conversion of Predehl \& Schmitt 1995 , assuming $R_{V}=3.1$ (Cardelli et al. 1989). Central densities are recalculated from the prescription of Djorgovski 1993. Numbers of sources from the quoted X-ray analyses inside the cluster half-mass radii, with luminosities in the $0.5-2.5 \mathrm{keV}$ range. NS refers to all accreting NS systems, including transients in NGC 6440 and Terzan 5. Close encounter rate $\Gamma$ is calculated by $\Gamma \propto \rho_{0}^{1.5} r_{c}^{2}$, as a percentage of the total Galactic globular cluster system rate.

${ }^{a}$ The letter $\mathrm{c}=$ core collapsed. NGC 6752 (Lugger, Cohn, \& Grindlay 1995) and NGC 6266 (Harris 1996) may not be core collapsed.

${ }^{\mathrm{b}}$ Including subtraction of probable background sources, based on Giacconi et al. $2001 \log N-\log S$. We have subtracted one hard source from NGC 6266, two from 47 Tuc, one from M28, five from $\omega$ Cen, and two from NGC 5904.

References.- (1) Pooley et al. 2002b; (2) this work; (3) Cohn et al. 2002; (4) Heinke et al. 2003; (5) Pooley et al. 2003a; (6) D. Pooley et al. 2003, in preparation; (7) Grindlay et al. 2001a; (8) C. O. Heinke et al. 2003, in preparation; (9) Heinke et al. 2003b; (10) Becker et al. 2003; (11) Pooley et al. 2002a; (12) Rutledge et al. 2002a; (13) Cool et al. 2002; (14) Gendre et al. 2003a; (15) van Leeuwen \& Le Poole 2002; (16) P. M. Lugger et al. 2003, in preparation; (17) Grindlay et al. 2001b; (18) Anthony-Twarog \& Twarog 2000; (19) Sosin 1997; (20) Webb et al. 2002; (21) Gendre et al. 2003b; (22) Verbunt 2001; (23) C. Bassa et al. 2003, in preparation.

Since we are quoting true radii and $T_{\text {eff }}$ rather than $R^{\infty}$ and $T_{\text {eff }}^{\infty}$, we calculate the temperature and radius results using the assumption of $z=0.306$ (appropriate for a $10 \mathrm{~km}, 1.4$ $M_{\odot}$ NS). The luminosity values given in Gendre et al. (2003b) and Becker et al. (2003) are not for the same energy ranges as our $0.5-2.5 \mathrm{keV}$ range. We used the Zavlin et al. (1996) $n s a$ model, with the same temperatures and radii as the best fits in these papers, to calculate the ratios of unabsorbed flux in our energy bands to their energy bands, and thus generated the luminosities in Table 2.

\section{3. $\omega$ Cen, NGC 6397, Terzan 5, and M80}

For these clusters we reextract source and background spectra of qLMXB candidates in the same way as in the original works (Rutledge et al. 2002a; GHE01b; Heinke et al. 2003a, 2003b), except that we use an extraction circle of $2^{\prime \prime}$ radius for NGC $6397 \mathrm{U} 24$. We calculate the fraction of the $1 \mathrm{keV}$ Chandra point-spread function that is included in each region using the CIAO tool mkpsf. We apply a correction to the effective area functions ${ }^{6}$ to account for the degradation of low-energy quantum efficiency. We group the channels to $\geq 20$ counts per bin to ensure applicability of the $\chi^{2}$ statistic and fit the data in XSPEC. For M80 CX6 (only 62 counts), we do not group the channels and instead use the C-statistic for analysis. This would be preferable for the faint sources in Terzan 5, but the background is extremely high because of the outburst by the transient LMXB. When

\footnotetext{
${ }^{6}$ Using the ACISABS model; see http://cxc.harvard.edu/cal/Acis/Cal_prods/qeDeg.
}

we use the C-statistic, we test the fit by generating Monte Carlo simulations of the best-fit spectrum. Roughly half the Monte Carlo simulations should show lower values of the $\mathrm{C}$-statistic than the data if the fit is good. The percentage of simulations showing a lower $\mathrm{C}$-statistic than the best fit is shown in Table 2 for sources where we use the $\mathrm{C}$-statistic.

For each qLMXB we fit a model consisting of photoelectric absorption fixed at the cluster value and the nonmagnetic hydrogen atmosphere of Lloyd (2003) plus an optional power-law component. The power-law component in field systems has been observed with a photon index ranging from 1 to 2 and contributing up to $40 \%$ of the flux (see Rutledge et al. 2002b, but cf. Campana et al. 2002). To simplify our analysis and allow direct comparison between objects, we fix the photon index of the possible power-law component at 1.5. The results of these fits are shown in Table 2. Luminosities are increased to account for aperture corrections by factors of 1.10 for Terzan 5, 1.02 for $\omega$ Cen, 1.03 for NGC 6397, and 1.07 for M80; derived radii are adjusted accordingly. All spectral errors, but not luminosity errors, are given at $90 \%$ confidence for a single parameter using the XSPEC error command. For power-law component upper limits, this translates to $95 \%$ confidence. We note that this procedure may significantly underestimate strongly covariant uncertainties, such as radius and temperature, especially when the absorption is large (see in 't Zand et al. 2001). Therefore we offer radius estimates as a consistency check, not as a serious attempt to constrain the radii of these objects, especially for small numbers of counts. This is particularly true since we have not considered intrinsic absorption in these systems. The values for Terzan 5 are 
TABLE 2

Probable qLMXBs in Globular Clusters

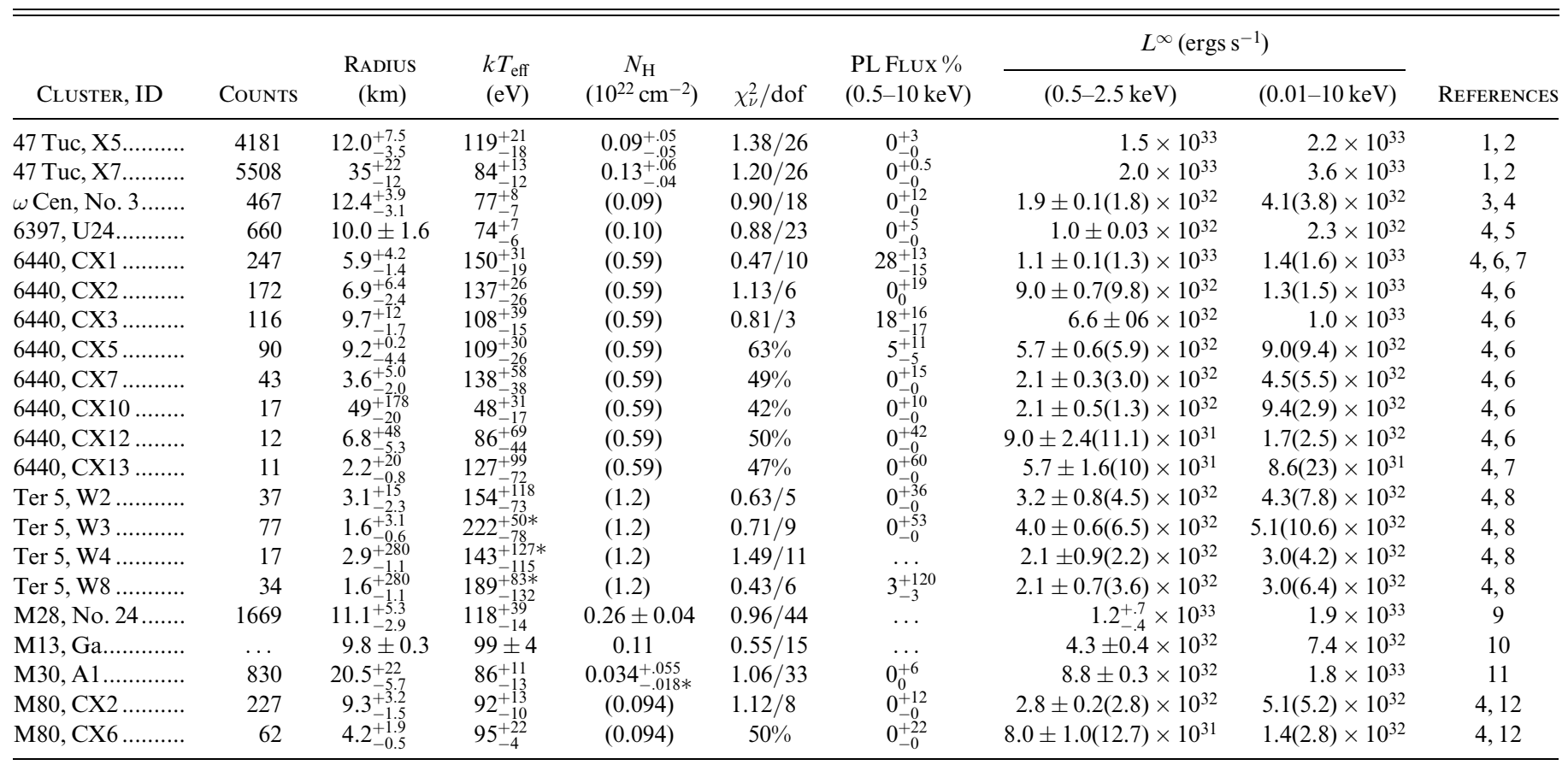

Nоте.-Parameters arederived from spectral fits to probable qLMXBs in globular clusters. Spectral fits are in XSPEC, using models of Lloyd (2003; except for M28 and M13 [see text]) with gravitational redshift fixed to 0.306 (except for 47 Tuc, M30; see references). Photoelectric absorption is fixed at the known Galactic absorption to the cluster for the fainter sources and equal to or greater than the Galactic absorption for brighter sources. Parameter values for CX5, CX7, CX10, CX12, CX13 in 6440 and CX6 in M80 are derived using C-statistic in XSPEC. For C-statistic fits, the percentage of Monte Carlo simulations generating a C-statistic less than the best fit are given in place of the $\chi^{2}$ statistic. Luminosities are given from the best fit and, where the fit is reasonable, for a fit with radius forced to $10 \mathrm{~km}$ (in parentheses). All errors are $90 \%$ confidence limits on one parameter, except for X-ray luminosity errors, which are $1 \sigma$ errors derived from counting statistics, ignoring uncertainty in other parameters. An asterisk indicates that the fit encountered a hard limit. For M80 CX6, $N_{\mathrm{H}}$ must be freed to allow an acceptable fit with radius $=10 \mathrm{~km}$, giving $N_{\mathrm{H}, 22}=0.21_{-10}^{+.05}$.

References. - (1) Grindlay et al. 2001a; (2) Heinke et al. 2003c; (3) Rutledge et al. 2002a; (4) reanalyzed in this work; (5) Grindlay et al. 2001b; (6) Pooley et al. 2002b; (7) in 't Zand et al. 2001; (8) Heinke et al. 2003a; (9) Becker et al. 2003; (10) Gendre et al. 2003b; (11) P. M. Lugger et al. 2003, in preparation; (12) Heinke et al. 2003 b.

questionable because of the extremely high background. For the Terzan 5 source W4 a possible power-law component cannot be constrained. We include these parameters for completeness and to generate luminosities. Upcoming Chandra observations of Terzan 5 in 2003 July (PI: R. Wijnands) should allow much better constraints on these sources if the transient remains in quiescence as expected.

\subsection{NGC 6440}

For NGC 6440 we downloaded the data from the archive and removed the pixel randomization applied in standard processing. Then we extracted spectra from 1.!2 radius circles around wavdetect-identified locations of the softer sources (CX1, CX2, CX3, CX5, CX7, CX8, CX10, CX11, CX12, and CX13) from Pooley et al. (2002b), except where severe crowding reduced our region size (to 0 ".86 for CX11). We grouped counts to 20 per bin, except when the source had fewer than 100 counts, in which case we left the data unbinned and used the C-statistic in XSPEC for analysis (as described above). We fix the absorption to the cluster value of $0.59 \times 10^{22} \mathrm{~cm}^{-2}$ (as in Pooley et al. 2002b) for all sources. We demand that candidate qLMXBs be acceptably fitted with a hydrogen atmosphere alone or require that the power-law component comprise no more than $40 \%$ of the 0.5-10 keV flux (see above). All luminosities and radii are adjusted to account for the extraction regions; this amounts to a factor of 1.07 in luminosities for the qLMXBs that we identify. In this way, we find several additional qLMXB candidates in NGC 6440.

The quiescent spectrum of the X-ray transient NGC 6440 CX1 was analyzed by in 't Zand et al. (2001), who assume a higher $N_{\mathrm{H}}$ column $\left(0.82 \times 10^{22} \mathrm{~cm}^{-2}\right)$ than the Galactic column to the cluster. They base this on the agreement between the $N_{\mathrm{H}}$ columns derived by a 1998 BeppoSAX observation of CX1 in outburst (in't Zand et al. 1999) and their analysis of the spectrum of an annulus around the outbursting transient in a 2001 Chandra observation. However, the BeppoSAX spectrum gives different $N_{\mathrm{H}}$ values depending on the assumed model, and the Chandra 2001 annulus spectrum of the outburst will be affected by the energy dependence of the point-spread function. Also, internal absorption is often observed to vary in accreting systems, especially during outbursts. Therefore, we perform our own determination of $N_{\mathrm{H}}$ for the quiescent spectrum of CX1. When we allow $N_{\mathrm{H}}$ to float, we find $N_{\mathrm{H}, 22}=0.66_{-.10}^{+.11}$. Using the $n s a$ model of Zavlin et al. (1996) and forcing the power-law photon index to be 1.44 (as done in in 't Zand et al. 2001), but using more recent photoelectric cross sections, we find $N_{\mathrm{H}, 22}=0.70_{-.10}^{+.18}$ (and $R=9.5_{-1.5}^{+0.7} \mathrm{~km}$ ). We consider the absorption to $\mathrm{CX} 1$ to remain uncertain and quote results assuming the cluster absorption in Table 2.

Our results strongly support the suggestion by Pooley et al. (2002b) that CX2, CX3, CX5, and CX7 are likely 
qLMXBs. Although the best fits for CX3 and CX5 include a power-law component, this component cannot be considered significantly detected. We also identify three additional possible qLMXBs, CX10, CX12, and CX13. Each of these objects is consistent with a $10 \mathrm{~km}$ hydrogen-atmosphere NS spectrum, without a power-law component. Their luminosities are also similar to those of known qLMXBs in $\omega$ Cen (Rutledge et al. 2002a), NGC 6397 (GHE01b), and M80 (CX6; Heinke et al. 2003b). None of these objects show clear variability, although the number of counts from each is low. We include their spectral parameters in Table 2, although we note that their low counts make these parameters individually unreliable.

For the harder objects CX8 and CX11, we can rule out a hydrogen atmosphere as the primary contribution to the observed flux. For CX8 a $10 \mathrm{~km}$ radius hydrogen atmosphere is a bad fit (100 Monte Carlo simulations of the best fit gave uniformly lower values of the C-statistic than the data). A hydrogen atmosphere with an additional powerlaw component is an acceptable fit but has $70_{-24}^{+30} \%$ of the unabsorbed $0.5-10 \mathrm{keV}$ flux ( $92 \%$ of the received flux) in the power-law component. For CX11 a $10 \mathrm{~km}$ radius hydrogen atmosphere alone is as bad a fit as for CX8. A hydrogen atmosphere with an additional power-law component is an acceptable fit but has $47_{-24}^{+30 \%}$ of the unabsorbed $0.5-10 \mathrm{keV}$ flux ( $83 \%$ of the received flux) in the power-law component. Although the uncertainties of the fit allow less than $40 \%$ of the unabsorbed flux to be in the power-law component, we think this object is unlikely to be a qLMXB. The remaining sources above CX11's luminosity are too hard to fit our canonical qLMXB model.

Fainter sources in the observation are uniformly harder than our suggested qLMXBs and have too few counts for even the simplest spectral fitting to be meaningful. We note that CX13 is as faint as or fainter than the faintest other qLMXBs identified in any globular cluster. Although confusion may have prevented the identification of perhaps one qLMXB, we conclude that this census of the qLMXBs in NGC 6440 is essentially complete. Follow-up observations of NGC 6440 scheduled for 2003 July (PI: R. Wijnands) will allow testing of these results.

Finally, we utilize a simple graphical method to check the similarity of our candidate qLMXBs to other qLMXBs. We place each of the NGC 6440 qLMXB candidates on a standardized X-ray CMD, using our best-fit luminosities and the unreddened colors derived by Pooley et al. (2002b). This standardized X-ray CMD (see GHE01a; Pooley et al. 2002a; Becker et al. 2003; Heinke et al. 2003a, 2003b) uses the unabsorbed luminosity in the $0.5-6 \mathrm{keV}$ band as the $y$-axis and 2.5 times the logarithm of the ratio of the numbers of counts in the $0.5-1.5$ and $1.5-6 \mathrm{keV}$ bands as the $x$-axis (or Xcolor), adjusted to remove the effects of reddening (Fig. 1). We also calculate the positions of several other qLMXBs in other clusters on the same diagram. To do this requires compensating for the reddening to different clusters, as well as accounting for the differences between the response of the front-illuminated ACIS-I chips (used for the observations of 47 Tuc, NGC 6397, $\omega$ Cen, and Terzan 5 ) and the back-illuminated ACIS-S chip S3. We used the CIAO PIMMS tool to estimate the difference in observed Xcolor for several spectra between each cluster's actual observation and a hypothetical ACIS-S observation with no reddening. We derive Xcolor offsets of +0.70 for $\omega$ Cen, +0.49 for 47 Tuc, +0.73 for NGC 6397 , and +0.06 for M30

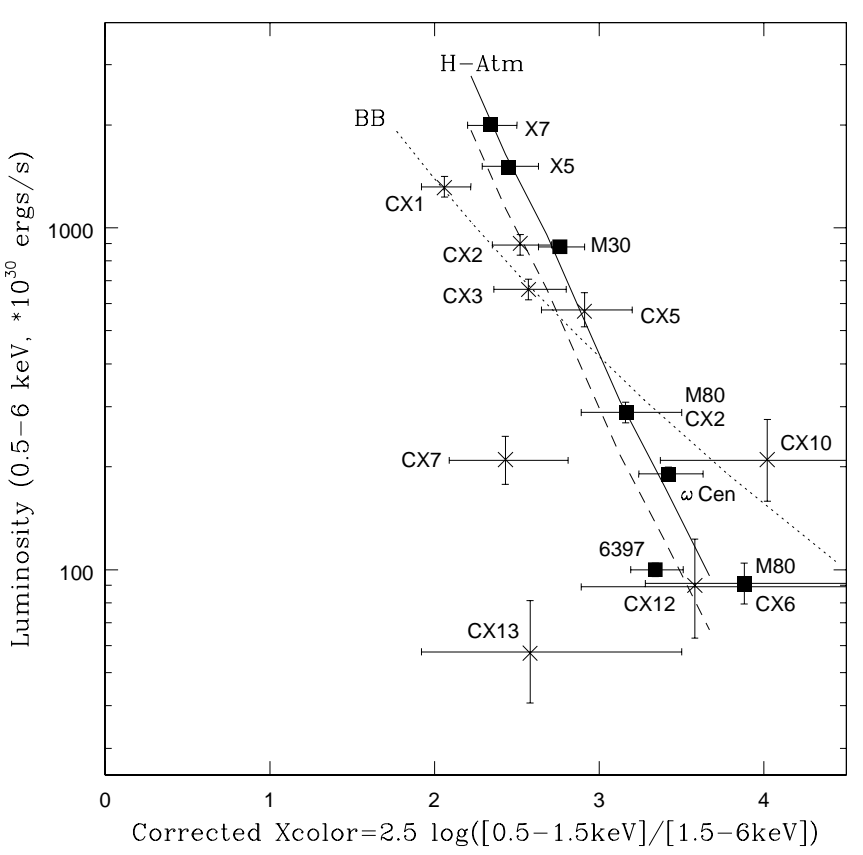

Fig. 1.-Standardized X-ray CMD with several qLMXBs and theoretical cooling tracks plotted. Crosses represent NGC 6440 probable qLMXBs, while squares represent qLMXBs from other clusters (NGC 6397, $\omega$ Cen, M30, M80 CX2 and CX6, and 47 Tuc X5, X7). A theoretical $1.44 \mathrm{~km}$ blackbody track is plotted (dotted line), as are 10 (dashed line) and $12 \mathrm{~km}$ (solid line) nonmagnetic hydrogen-atmosphere models from Lloyd (2003). Errors are from Gehrels (1986), applied to the counts detected in each band, and do not include possible errors in the best-fit luminosity from spectral fitting. CVs (not shown) tend to have corrected Xcolors near zero on this scale (see GHE01a; Cool et al. 2002; Heinke et al. 2003c).

to place their qLMXBs onto a dereddened ACIS-S CMD. The two qLMXBs in 47 Tuc suffer moderate pileup, which distorts their intrinsic Xcolor. To reduce the hardening effect of pileup, we use only the Xcolor derived from the final 200047 Tuc observation ( $4.7 \mathrm{ks}$ ), which used a $\frac{1}{4}$ subarray to avoid pileup (receiving 369 and 423 counts for X5 and $\mathrm{X} 7$, respectively). However, we note that these objects (and the M30 qLMXB) still suffer pileup at the $~ 2 \%$ level, which will harden their spectra and is not accounted for. The Terzan 5 qLMXBs suffer extremely high background levels, as well as the highest reddening, so we leave their relatively poor-quality data off this plot. The loss of low-energy $(<1 \mathrm{keV})$ sensitivity over the Chandra mission has a minor effect on the observed Xcolors, creating a maximum shift of 0.3 in Xcolor if responses from the latest observation discussed (M30) are not corrected for this continuous degradation. All but two of these observations (M80 and M30, nearly contemporaneous) either were taken with the ACIS-I array (with less low-energy sensitivity) or suffered very high galactic absorption. In either case, the effects of the low-energy quantum efficiency degradation are significantly reduced.

We plot the theoretical locations in this diagram of hydrogen atmosphere NSs of 10 and $12 \mathrm{~km}$ radii over a range of temperatures, using the models of Lloyd (2003) with the gravitational redshift fixed to $z=0.306$ and response files from 2001 October (epoch of the M80 observation). These are essentially NS cooling tracks. This X-ray CMD clearly shows agreement between the theoretical hydrogen atmosphere cooling tracks and the observed 
locations of identified and candidate qLMXBs. We caution that the apparent close agreement with the $12 \mathrm{~km}$ track should not be taken too seriously given the numerous systematic errors listed above. We also plot the theoretical track of a blackbody, with radius arbitrarily set to $1.41 \mathrm{~km}$. A clear difference can be seen between the predictions of a blackbody versus the hydrogen atmosphere models, with the colors and luminosities of the qLMXBs favoring the hydrogen atmosphere model. This diagram shows the utility of a standardized X-ray CMD and the importance of hydrogen-atmosphere NS models.

\subsection{Other Clusters}

Several other clusters have been studied with Chandra's ACIS detector to a depth sufficient to identify any of the qLMXBs discussed above. Pooley et al. (2002a) find no objects in NGC 6752 with luminosities and spectra similar to those found in other clusters. However, they do identify a source (CX8) within the half-mass radius of NGC 6752 that shows an extremely soft spectrum, placing it near the NS cooling track in Figure 1, at an implied $L_{\mathrm{X}}=2 \times 10^{31} \mathrm{ergs}$ $\mathrm{s}^{-1}$. We note that no thermally radiating MSP has been identified at these luminosities; from Grindlay et al. (2002), we see that the $0.5-2.5 \mathrm{keV}$ X-ray luminosities of thermally emitting MSPs are in the range (1-4) $\times 10^{30} \mathrm{ergs} \mathrm{s}^{-1}$. Nonthermally emitting MSPs can be brighter (GHE01b; Edmonds et al. 2002a; Becker et al. 2003) but also have much harder spectra. Identification of a thermally emitting NS in the "gap" between the tail of the qLMXB luminosity function and the MSP nexus would be of great interest.

We extracted the spectrum of CX8 from the Chandra archival observation of NGC 6752, using a $2^{\prime \prime}$ extraction region. We fitted the data using the C-statistic, since there are only 83 counts. XSPEC fits with a hydrogen atmosphere model give atrocious fits. Fixing the radius to $10 \mathrm{~km}$ leads to $100 \%$ of simulations producing a lower C-statistic, whether the $N_{\mathrm{H}}$ is fixed to the cluster value or not. Allowing the radius to be a free parameter allows a marginal but acceptable fit ( $84 \%$ of simulations lower than the derived Cstatistic), but the radius is reduced to $1.0_{-0.3}^{+0.5} \mathrm{~km}$. (We check these results by binning to 10 counts bin $^{-1}$ and using the $\chi^{2}$ statistic, deriving similar results.) The poor quality of these fits is induced by a feature resembling an emission-line complex located at $\sim 0.9 \mathrm{keV}$. This suggests an optically thin low-temperature plasma, so we fit an XSPEC MEKAL optically thin plasma model (Liedahl, Osterheld, \& Goldstein 1995 and references therein), deriving $k T=$ $0.77 \pm .11 \mathrm{keV}$ and an iron abundance $21_{-10}^{+17 \%} \%$ of solar. This iron abundance is inconsistent with the metallicity of NGC 6752, $[\mathrm{Fe} / \mathrm{H}]=-1.65$ (3\% solar), from Harris (1996). The metallicity inconsistency and the large $\left(\sim 88^{\prime \prime}, 8.5\right.$ core radii) offset of CX8 from the core of NGC 6752 indicate that CX8 may not be a member of the cluster but rather a foreground star or active binary.

Preliminary analyses of deep Chandra or XMM data have been presented for five other clusters at scientific conferences or in other works at the time of writing. According to both D. Pooley et al. (2003, in preparation ) and Gendre et al. (2003b), NGC 6366 possesses no qLMXBs. NGC 6121 has no qLMXBs (C. Bassa et al. 2003, in preparation); nor does NGC 5904 (D. Pooley et al. 2003, in preparation). The rich, dense cluster NGC 6266 (Pooley et al. 2003) has five identified qLMXBs (D. Pooley 2003, in preparation).
Reassessment of the $X M M$ data on M22 (Webb et al. 2002) by Gendre et al. (2003b) indicates that no qLMXBs matching our template are present in that cluster. In each case the sensitivity of the observation is sufficient to confidently identify all possible qLMXBs above our empirical lower $L_{\mathrm{X}}$ limit of $1 \times 10^{32} \mathrm{ergs} \mathrm{s}^{-1}$. In most of these clusters the sensitivity is sufficient to identify all harder sources above $L_{\mathrm{X}}(0.5-2.5 \mathrm{keV})>10^{31} \mathrm{ergs} \mathrm{s}^{-1}$ (Pooley et al. 2003; see Table 1). We use these preliminary results in our analysis of cluster weightings in $\S 3.3$.

\section{COMPARATIVE ANALYSIS}

\section{1. qLMXB Spatial Distribution}

Grindlay et al. (1984) estimated the typical mass of the bright LMXBs in eight globular clusters by analyzing the distribution of the radial offsets of these sources relative to the cluster centers. This approach assumes that the sources have a common mass and that the cluster potentials have a common structure. It is also assumed that the distributions of X-ray sources and normal stars are in thermal equilibrium. In this case, the most massive group of normal stars is expected to be approximately distributed as a King (1966) model. This analysis results in an estimate for the ratio $q=M_{\mathrm{X}} / M_{*}$ of the source mass to the mass of the typical star that defines the optical core radius.

Using a maximum likelihood analysis, Grindlay et al. (1984) found a most likely value of $q=2.6$ with a $90 \%$ confidence range of 1.8-3.8. In this section we adapt this analysis to the qLMXB distribution, in order to estimate the qLMXB mass.

Grindlay et al. (2002) describe a maximum likelihood approach for fitting "generalized King models" to the projected radial distributions of cluster objects. In this model, the projected surface density of each component is described by

$$
S(r)=S_{0}\left[1+\left(\frac{r}{r_{0}}\right)^{2}\right]^{\alpha / 2},
$$

where $\alpha$ is the power-law index and the core radius $r_{c}$ is related to the radial scale parameter $r_{0}$ by $r_{c}=$ $\left(2^{-2 / \alpha}-1\right)^{1 / 2} r_{0}$. Grindlay et al. (2002) obtained independent estimates of $r_{c}$ and $\alpha$ for various source populations in 47 Tuc by maximum likelihood fits of equation (1). If the optical core radius $r_{c *}$ of the cluster is defined by stars of mass $M *$ that have a standard King model distribution $\left(\alpha_{*}=-2\right)$, then the core radius and slope for the distribution of sources of mass $M_{X}=q M_{*}$ are related to $q$ by

$$
\begin{aligned}
r_{c X} & =\left(2^{2 /(3 q-1)}-1\right)^{1 / 2} r_{c *}, \\
\alpha_{X} & =1-3 q .
\end{aligned}
$$

In the present study we first explored two-parameter maximum likelihood fits of equation (1) to the distribution of 20 qLMXBs in seven clusters with King model structure (47 Tuc, NGC 6440, Terzan 5, M80, M28, $\omega$ Cen, and M13note that we include the Terzan 5 transient LMXB). However, this produced large uncertainty ranges for both $r_{c X}$ and $\alpha_{X}$ and thus did not provide much useful information on the qLMXB mass. We then performed single-parameter fits by determining the value of $q$ that maximizes the likelihood of observing the given sample, with $r_{c X}$ and $\alpha_{X}$ given 
by equation (2). Thus, we fit the function

$$
S(r)=S_{0}\left[1+\left(\frac{r}{r_{c *}}\right)^{2}\right]^{(1-3 q) / 2}
$$

This is similar to the approach of Grindlay et al. (1984). We estimate the uncertainty range for $q$ by fitting 1000 bootstrapped resamples of the original 20 source sample (see Cohn et al. 2002). The result is $q=1.9 \pm 0.2(1 \sigma)$ with a $90 \%$ confidence range of $1.6 \leq q \leq 2.2$. The corresponding values of core radius and slope are $r_{c X}=(0.60 \pm 0.04) r_{c} *$ and $\alpha_{X}=-4.5 \pm 0.5$.

We note that the assumption of thermal equilibrium among the cluster objects is not strictly justified for $\omega$ Cen, which has not reached equipartition (Anderson 1997). Therefore, we removed the qLMXB in $\omega$ Cen from our sample, repeated the analysis, and derived the same results. We performed the same analysis on the sample of eight LMXBs used in Grindlay et al. (1984), finding $q=2.2$ with $90 \%$ confidence range of $1.7 \leq q \leq 3.7$. This is in reasonable agreement with their determination of $q=2.6,1.8 \leq q \leq 3.8$ (90\% confidence), with the major difference being that the current analysis does not consider offset measurement errors (negligible for Chandra positions). Finally, we performed the same analysis on the sample of soft X-ray sources in 47 Tuc from Grindlay et al. (2002), differing from that analysis by parameterizing both the core radius and power-law slope $\alpha$ in terms of a single $q$. Our new value for these soft sources is $q=1.58 \pm 0.13(90 \%$ confidence range $1.40 \leq q \leq 1.81$ ).

If we assume that the optical profiles of the sample clusters, from which the core radii were determined, are dominated by turnoff-mass objects, then a reasonable estimate for $M_{*}$ is $0.8 M_{\odot}$ (see, e.g., King et al. 1998). This results in a most likely qLMXB mass of $M_{X}=(1.5 \pm 0.2) M_{\odot}$ with a $90 \%$ confidence range of $1.3 M_{\odot} \leq M_{\mathrm{X}} \leq 1.8 M_{\odot}$. This range comfortably allows for a Chandrasekhar-mass NS with a low-mass companion. For the soft sources in 47 Tuc (which probably include some ABs as well as MSPs; Edmonds et al. 2003b), we derive a mass range of $M_{X}=1.26 \pm 0.10 M_{\odot}$, with $90 \%$ confidence range $1.12 M_{\odot} \leq M_{X} \leq 1.45 M_{\odot}$. For the eight LMXBs from Grindlay et al. (1984), our $90 \%$ confidence range is $1.4 M_{\odot} \leq M_{X} \leq 3.0 M_{\odot}$. Both of these estimates are also consistent with the qLMXB range.

\subsection{Luminosity Functions}

Recent work by Pooley and collaborators (Pooley et al. 2002b; D. Pooley et al. 2003, in preparation) has shown clear differences between luminosity functions (LFs) of different clusters, attributed in large part to differences in source makeup (e.g., large numbers of MSPs and ABs in 47 Tuc compared with NGC 6397). The LFs of globular cluster $\mathrm{X}$-ray sources should be affected both by the relative numbers of different sources in each cluster and by the properties of the individual populations. Here we make a first attempt to characterize the LFs for two identifiable source populations, qLMXBs and harder sources with $L_{X}>10^{31} \mathrm{ergs} \mathrm{s}^{-1}$, identified as mostly CVs (see $\S 2$ ). For the latter group, we test whether the hard source luminosity functions in different clusters are consistent, in order to identify possible differences between the $\mathrm{CV}$ populations of different clusters.
To study the luminosity distributions of these populations, we follow the formalism of Johnston \& Verbunt (1996) and Pooley et al. (2002a). Because of the relatively small numbers of sources, we assume for this analysis a simple power-law shape for the LF above a limiting luminosity. We derive the best-fit luminosity function by forming the quantities $z_{j}=\left(L_{i}^{j} / L_{i}\right)^{-\gamma}$ and finding the value of $\gamma$ that most uniformly distributes the $z_{j}$ along the interval $[0,1]$. Here $i$ indicates the cluster, $j$ indicates the source, $L_{i}^{j}$ is the source luminosity, and $L_{i}$ is the limiting luminosity to which the cluster has been searched, or the minimum luminosity of the analysis. If the true LF is not a power law, using differing limiting luminosities for different clusters will generate apparent differences between LFs of different clusters. Therefore we attempt to use the same limiting luminosity for each cluster within one analysis. For qLMXBs we include all qLMXBs in $\S 2$, analyzing both the luminosities generated with $10 \mathrm{~km}$ fits and those where the radius was allowed to float. We take as our minimum luminosity the lowest luminosity of a detected qLMXB. This leaves only Terzan 5 with a higher limiting luminosity, as we could detect qLMXBs with lower luminosities in each of the other clusters. We perform our qLMXB analysis both with and without the (lower signal-to-noise ratio) Terzan 5 data, using the faintest qLMXB detected in Terzan 5 as its limiting luminosity. The results of this analysis are listed in Table 3, and the cumulative qLMXB luminosity function for all clusters except Terzan 5 is shown in Figure 2.

For the harder, primarily CV sources above $10^{31} \mathrm{ergs} \mathrm{s}^{-1}$, we use the luminosities reported in GHE01a for 47 Tuc, in Pooley et al. (2002a) for NGC 6752, and in Heinke et al. (2003b) for M80. For NGC 6397, we have adjusted the

TABLE 3

LUMINOSITY FUnCTION FITS

\begin{tabular}{|c|c|c|c|c|}
\hline Description & $L_{\min }$ & $\begin{array}{c}\text { Number of } \\
\text { Sources }\end{array}$ & $\gamma$ & $\begin{array}{c}\text { Probability } \\
(\%)\end{array}$ \\
\hline \multicolumn{5}{|c|}{ qLMXBs } \\
\hline$L_{\mathrm{X}}, R$ fixed .......... & $1.0 \times 10^{32}$ & 17 & $0.64_{-22}^{+.31}$ & 81 \\
\hline With Ter $5 \ldots . .$. & $1.0 \times 10^{32}$ & 21 & $0.76_{-.30}^{+.36}$ & 90 \\
\hline$L_{\mathrm{X}}, R$ free $\ldots \ldots \ldots \ldots$ & $5.7 \times 10^{31}$ & 17 & $0.46_{-.10}^{+.15}$ & 49 \\
\hline$L_{\text {Bol }}, R$ fixed ........ & $2.3 \times 10^{32}$ & 17 & $0.77_{-.29}^{+.43}$ & 91 \\
\hline With Ter $5 \ldots . .$. & $2.3 \times 10^{32}$ & 21 & $0.94_{-.41}^{+.48}$ & 93 \\
\hline \multicolumn{5}{|c|}{ Harder Sources } \\
\hline 47 Tuc only ........ & $1 \times 10^{31}$ & 18 & $0.85_{-.37}^{+.61}$ & 99 \\
\hline M80 only ............ & $1 \times 10^{31}$ & 14 & $0.65_{-.30}^{+.51}$ & 99 \\
\hline M28 only ............ & $1 \times 10^{31}$ & 14 & $0.79_{-.24}^{+.64}$ & 66 \\
\hline N6752 only ......... & $1 \times 10^{31}$ & 8 & $0.62_{-27}^{+.57}$ & 85 \\
\hline N6397 only ......... & $1 \times 10^{31}$ & 8 & $0.42_{-.13}^{+.29}$ & 54 \\
\hline Five clusters ........ & $1 \times 10^{31}$ & 62 & $0.67_{-.12}^{+.20}$ & 80 \\
\hline Six clusters .......... & $5 \times 10^{31}$ & 25 & $0.82_{-.15}^{+.19}$ & 42 \\
\hline
\end{tabular}

Note.-Results of K-S tests for the LFs of source populations in several globular clusters. For qLMXBs, sources include those in Table 2 omitting the four Terzan 5 sources, except where indicated. For harder sources, the five clusters are those listed individually, with NGC 6440 added to study only the bright CVs (six clusters). The quantity $L_{\min }$ is the limiting luminosity of each analysis (except for Terzan 5 qLMXBs, where $L_{X \text {, min }}=2 \times 10^{32}$ and $4 \times 10^{32}$ ergs s$^{-s 1}$ for $X$-ray and bolometric luminosities, respectively); $\gamma$ is the index of the best-fit LF $\left(d N / d L_{\mathrm{X}} \propto L_{\mathrm{X}}^{-(\gamma+1)}\right)$, and the listed errors are the range where the K-S probability is larger than $10 \%$ (where the K-S probability is the probability that a random sample selected from the given distribution will have a larger statistic). 


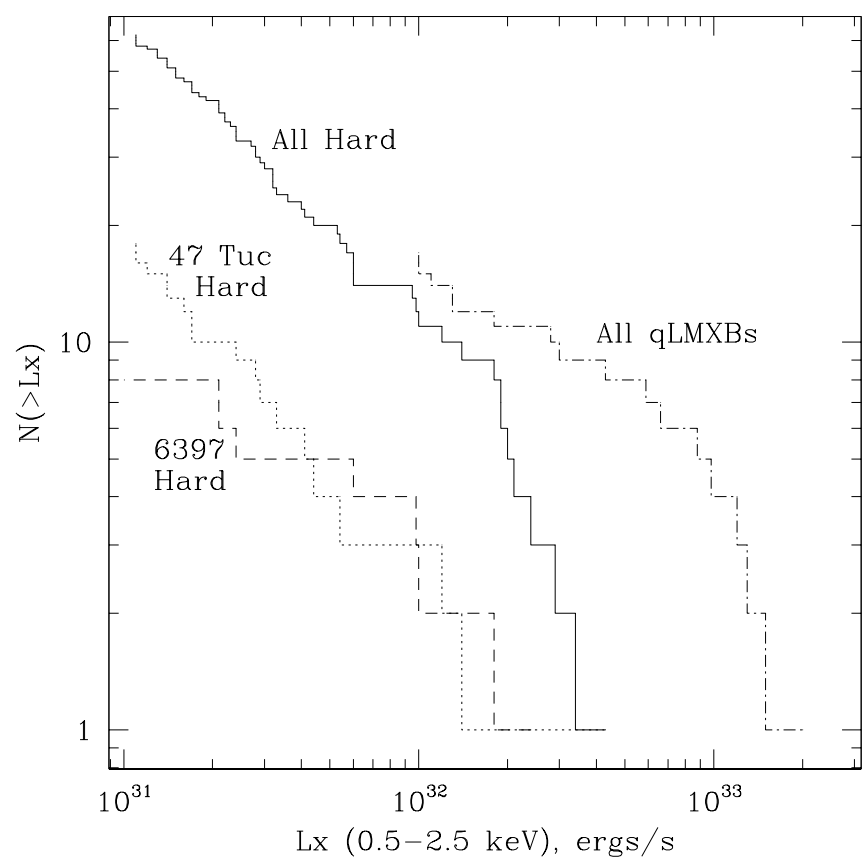

FIg. 2.-Cumulative LFs of qLMXBs and probable CVs in globular clusters. The qLMXBs are from Table 2, using values of $L_{\mathrm{X}}$ derived from fits assuming $10 \mathrm{~km}$ radius (where acceptable). The probable $\mathrm{CVs}$ are hard sources with $10^{31} \mathrm{ergs} \mathrm{s}^{-1}<L_{\mathrm{X}}(0.5-2.5)<10^{33} \mathrm{ergs} \mathrm{s}^{-1}$, with luminosities from 47 Tuc (GHE01a), NGC 6397 (GHE01b), NGC 6752 (Pooley et al. 2002a), M28 (Becker et al. 2003), and M80 (Heinke et al. 2003b). A few known active binaries have not been removed from the CV sample, but the bright MSP in M28 has been removed. One or two background AGNs are also expected among these sources.

luminosities calculated in GHE01b for a distance of $2.7 \mathrm{kpc}$ (Anthony-Twarog \& Twarog 2000). For M28, we calculated the $0.5-2.5 \mathrm{keV}$ unabsorbed X-ray luminosities of sources within the half-mass radius of the cluster from the reported 0.5-8.0 keV fits using PIMMS and the spectral fits in Becker et al. (2003). We use the MEKAL fits reported for M28 sources 17, 25, and 28 and the power-law fit for the remaining 11 sources (excluding the MSP). We have not removed the five known active binaries from this distribution, but we have removed the bright MSP in M28. We have not subtracted possible background active galactic nuclei (AGNs), which should number no more than 1-2 among these sources. We show the cumulative LF for the hard sources in these five clusters in Figure 2, along with the LFs for the hard sources in 47 Tuc and NGC 6397 separately. We also analyze the combined LF above $5 \times 10^{31} \mathrm{ergs} \mathrm{s}^{-1}$ for these five clusters plus NGC 6440 (Pooley et al. 2002a), which suffers incompleteness and crowding at lower luminosities. Finally, we separately analyze the hard source LFs down to $10^{31} \mathrm{ergs} \mathrm{s}^{-1}$ for the five deep cluster observations (47 Tuc, NGC 6397, NGC 6752, M80, and M28). The results of these analyses are listed in Table 3, while some of the Kolmogorov-Smirnov (K-S) probabilities as a function of $\gamma$ are shown in Figure 3.

The index of the qLMXB luminosity function depends strongly on the chosen minimum luminosity. Assuming that the qLMXBs are $10 \mathrm{~km}$ objects, the best-fit X-ray luminosity slope above the minimum is $\gamma=0.6$, while the bolometric luminosity slope is slightly steeper. The LFs of the hard source population in NGC 6397 and 47 Tuc are

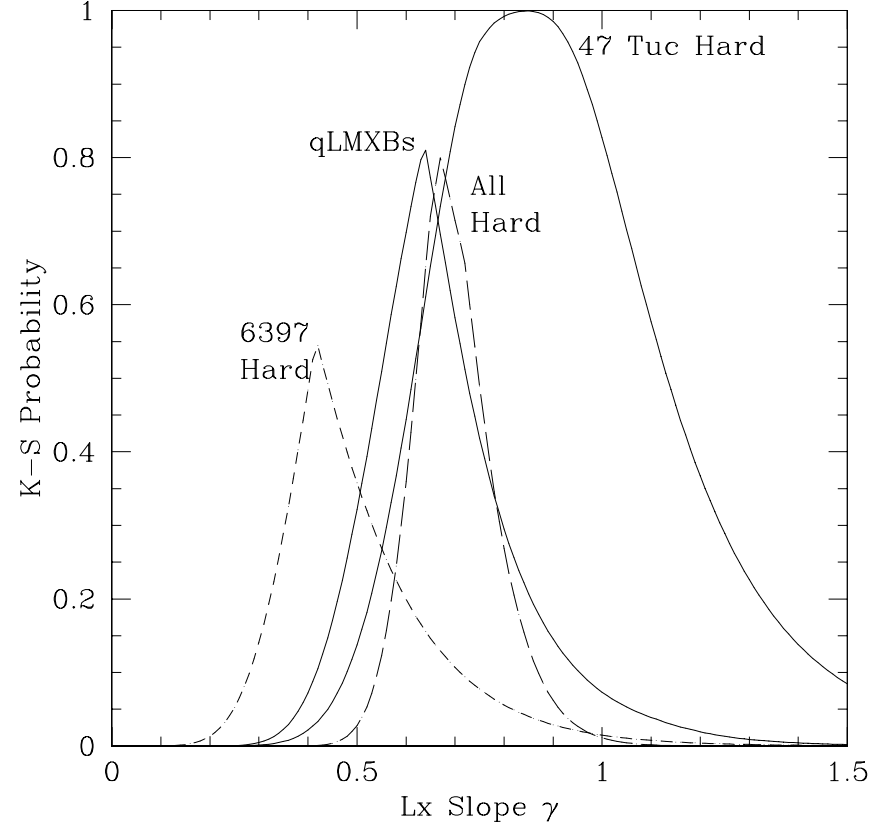

FIG. 3.-Comparison of the indices of power-law slopes for several different globular cluster populations. K-S probability is plotted as a function of $\gamma$, where the LF is assumed to be $d N \propto L_{\mathrm{X}}^{-\gamma} d \ln L_{\mathrm{X}}$. The qLMXB LF is that where qLMXB radii are assumed to be $10 \mathrm{~km}$, and Terzan 5 qLMXBs are excluded (first row in Table 3 ).

clearly distinct, and that of NGC 6397 differs from the other globular clusters in general (Figs. 2 and 3). Since CVs make up most of these hard sources, there must be a difference in the $\mathrm{CV}$ properties between these clusters. (Removing the three known ABs among the 47 Tuc sources and the one known $\mathrm{AB}$ among the 6397 sources does not affect the results.) We note that several cluster properties appear to correlate with the slope of the hard source LFs: cluster central density (inverse correlation), cluster metallicity, and cluster collision frequency $\Gamma$ (see Table 1). Since a large part of these correlations are due to the unusual cluster NGC 6397 (see $\S \S 4.3$ and 4.4), we do not attempt to draw conclusions about which properties are responsible for the different LF slopes.

Possible high-luminosity cutoffs may exist in the luminosity functions for both CVs and qLMXBs, as seen in Figure 2. We judge these cutoffs to be at roughly $L_{\mathrm{X}}(0.5-2.5 \mathrm{keV})=2 \times 10^{32} \mathrm{ergs} \mathrm{s}^{-1}$ for the CVs and $10^{33}$ $\mathrm{ergs} \mathrm{s}^{-1}$ for the qLMXBs. K-S tests show both samples to be formally consistent with power laws with no high- $L_{\mathrm{X}}$ cutoffs. However, the K-S probability decreases and $\gamma$ increases as the limiting luminosity increases, suggesting a cutoff. As the number of globular cluster X-ray sources identified in these luminosity ranges increases, it will become worthwhile to fit more complicated luminosity functions to the data.

\subsection{Dependence of X-Ray Source Numbers on Cluster Density and Core Radius}

We use the numbers of qLMXBs in several clusters of different structural parameters (all the clusters from Table 1) to attempt to constrain qLMXB and CV formation mechanisms. We assume that dynamical formation of globular cluster X-ray sources can be parameterized as 
$\Gamma=\rho_{c}^{\alpha} r_{c}^{\beta}$, where $\Gamma$ is the formation rate, $\rho_{c}$ is the central luminosity density, and $r_{c}$ is the core radius. We follow the density weighting method of Johnston \& Verbunt (1996) to test the dependencies of qLMXB formation on the exponents $\alpha$ and $\beta$. This method calculates the weight for each cluster on the basis of the choice of $\alpha$ and $\beta$ and assigns a line length proportional to that weight. The line length is reduced by the fraction of X-ray sources to our chosen limit that are detectable in each cluster. This affects only Terzan 5; using the estimate of $30 \%$ incompleteness above $10^{32} \operatorname{ergs~s}^{-1}$ (due to the LMXB; Heinke et al. 2003a), we find that $70 \%$ of the qLMXBs in Terzan 5 should have been detected, or $85 \%$ if the transient is included. The X-ray sources within each cluster are spread evenly along the line segment, and then the clusters are ordered by increasing weight to form a line of unit length with all the X-ray sources spread along it. A $\mathrm{K}-\mathrm{S}$ test then is applied to check whether the sources are consistent with a uniform distribution. The results of K-S tests for a range of values of $\alpha$ and $\beta$ are shown in Figure 4 , with the best-fit combination marked with a cross and $90 \%, 50 \%$, and $10 \%$ K-S probability contours marked. The meaning of a K-S probability $P$ is that a random distribution will be less uniform than the data $P \%$ of the time. However, since we distribute the sources evenly within each line segment, the K-S probabilities will be overestimates. Although the results are not extremely constraining, clearly $\alpha$ is best fit near a value of 1.5.

We perform the same analysis for harder sources above $10^{31}$ ergs s$^{-1}$ in a few clusters, which we believe are largely

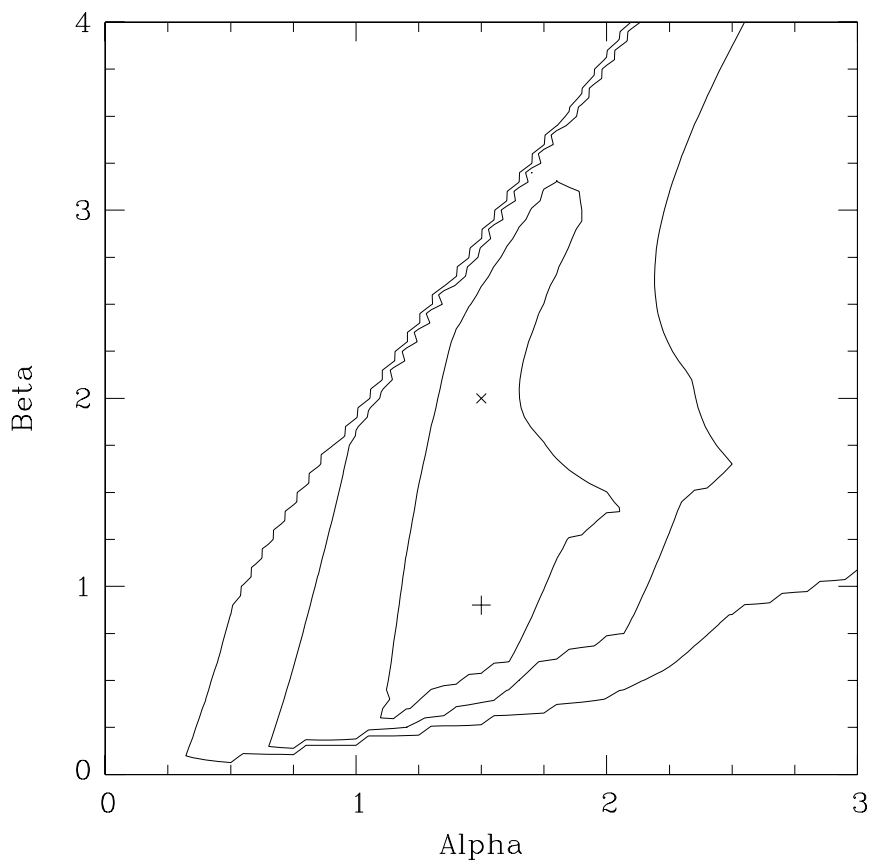

FIG. 4.-K-S probability contour map for the dependencies of qLMXB distribution among globular clusters on central cluster density $\rho_{0}$ and core radius $r_{c}$. The data are the numbers of accreting NSs (whether in outburst or quiescence) from all clusters in Table 1, plus an estimate of the incompleteness of the Terzan 5 survey (see text). We test the acceptability of a qLMXB formation rate $\Gamma$ of the form $\Gamma \propto \rho_{0}^{\alpha} r_{c}^{\beta}$ for various values of $\alpha$ and $\beta$. Contours indicate K-S probabilities of an acceptable distribution of $10 \%, 50 \%$, and $90 \%$, while the plus sign marks the best fit. The theoretically calculated close encounter rate dependency (Verbunt \& Hut 1987) is indicated by a cross.
CVs, and the subset of those sources above $10^{32} \mathrm{ergs} \mathrm{s}^{-1}$ in a larger range of clusters. To avoid dependence of our results on the assumed LF, we use data that are complete to our chosen limiting luminosity except for Terzan 5 , where the incompleteness to $10^{32} \mathrm{ergs} \mathrm{s}^{-1}$ is $30 \%$. For hard sources above $10^{32} \mathrm{ergs} \mathrm{s}^{-1}$, we use all the clusters in Table 1 except NGC 6366 (for which cluster membership is in doubt for all sources). For hard sources above $10^{31} \mathrm{ergs} \mathrm{s}^{-1}$, we exclude Terzan 5, NGC 6440, M13, and NGC 6366 because of their incompleteness. For $\omega$ Cen the results of Cool et al. (2002) and Gendre et al. (2003a) are in close agreement on the numbers of X-ray sources above $10^{31} \mathrm{ergs} \mathrm{s}^{-1}$ within the half-mass radius ( 15 vs. 16 ; source 9 is brightened in the $X M M$ data). This sample includes only one soft source, the qLMXB, so foreground stars do not seem to be a problem. We estimate 4.7 background AGNs from the analysis of Giacconi et al. (2001), and so estimate that nine hard sources above $10^{31} \mathrm{ergs} \mathrm{s}^{-1}$ belong to the cluster (at the time of the Chandra observation). We also subtract one expected background source from NGC 6266 and M28 and two expected background sources from 47 Tuc and NGC 5904, producing the numbers listed under "Hard Sources" in Table 1.

The results of these tests are shown in Figures 5 and 6 for the bright and full sample of hard sources, respectively. The hard sources require lower values of $\alpha$ than the qLMXBs, and the sources above $10^{31} \mathrm{ergs} \mathrm{s}^{-1}$ seem to require values of $\beta$ less than 2. This reduced dependence on the core radius may be traced to the large number of CVs in NGC 6397, which is a core-collapsed cluster with a very small core radius. We repeat the test for the full sample of hard sources, excluding NGC 6397, and find $\beta$ to be much more loosely constrained (Fig. 7). We discuss these results in $\S 4.3$.

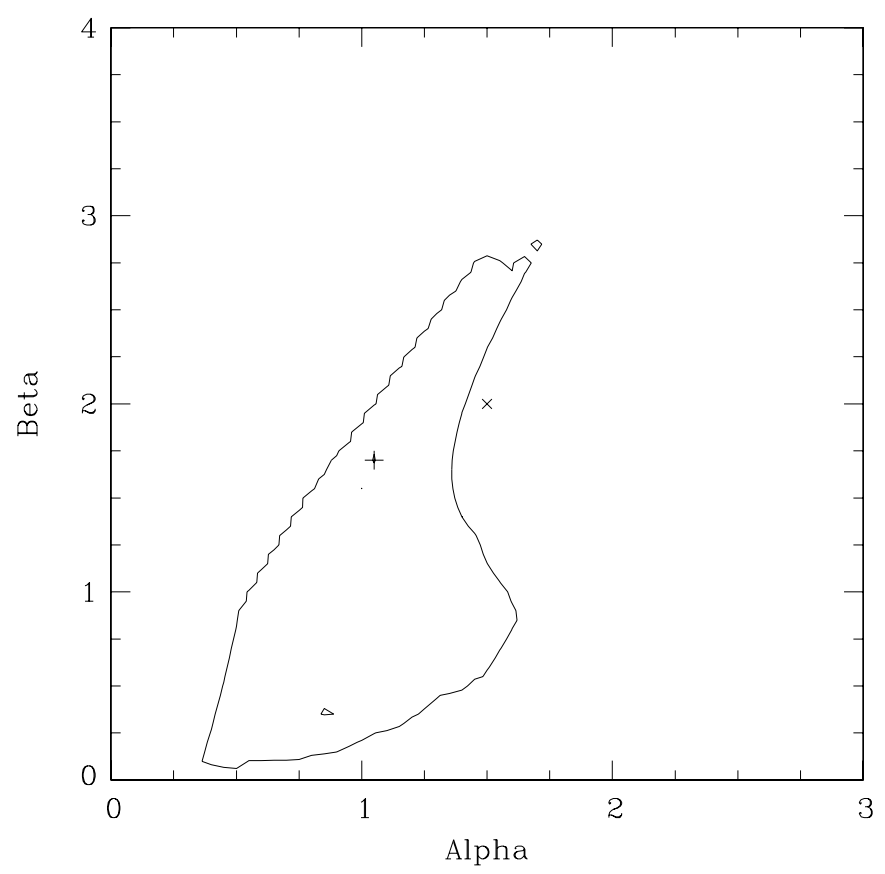

FIG. 5.-K-S probability contour map for the dependencies of hard globular cluster sources $\left[10^{32} \mathrm{ergs} \mathrm{s}^{-1}<L_{X}(0.5-2.5)<10^{33} \mathrm{ergs} \mathrm{s}^{-1}\right]$ on $\rho_{0}$ and $r_{c}$. Symbols and contours are as in Fig. 4, except that no $90 \%$ contour is indicated. Data are bright hard sources from all clusters in Table 1 except NGC 6366. 


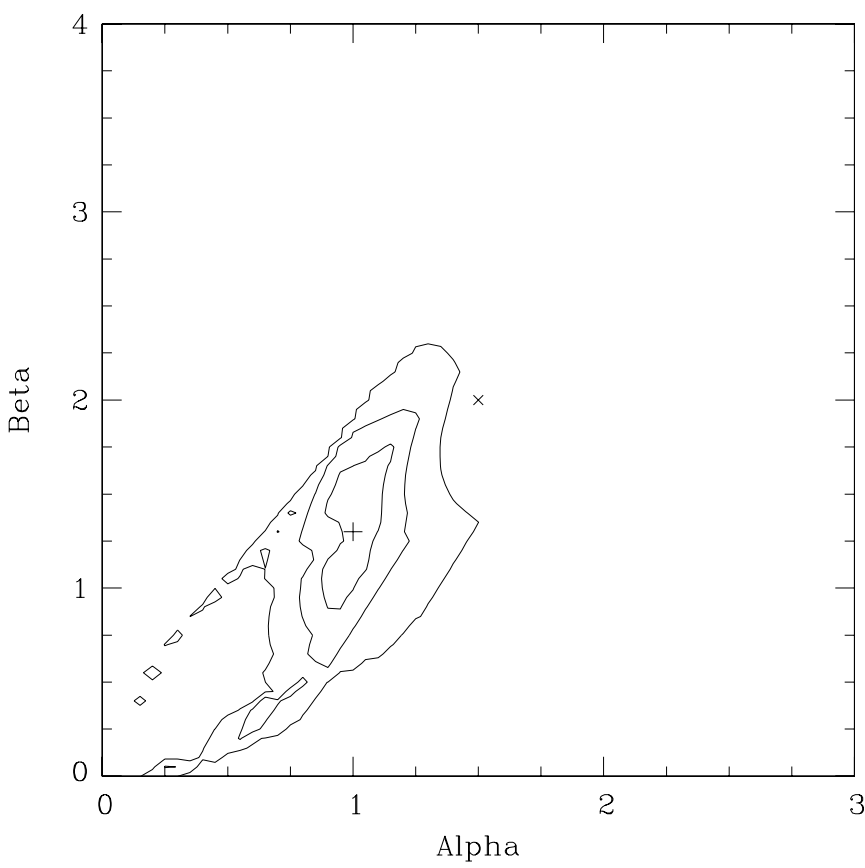

FIG. 6.-K-S probability contour map for the dependencies of hard globular cluster sources $\left[10^{31} \mathrm{ergs} \mathrm{s}^{-1}<L_{\mathrm{X}}(0.5-2.5)<10^{33} \mathrm{ergs} \mathrm{s}^{-1}\right]$ on $\rho_{0}$ and $r_{c}$. Symbols and contours are as in Fig. 4. Data are from Table 1, omitting Terzan 5, M13, NGC 6440, and NGC 6366.

\section{DISCUSSION}

\subsection{Spatial Distribution, Spectra, and Variability}

The spatial distribution of our qLMXB sample agrees with our expectation that these objects have masses characteristic of NSs plus low-mass companions. The implied average qLMXB system mass of $1.5_{-02}^{+0.3} M_{\odot}(90 \%$ confi-

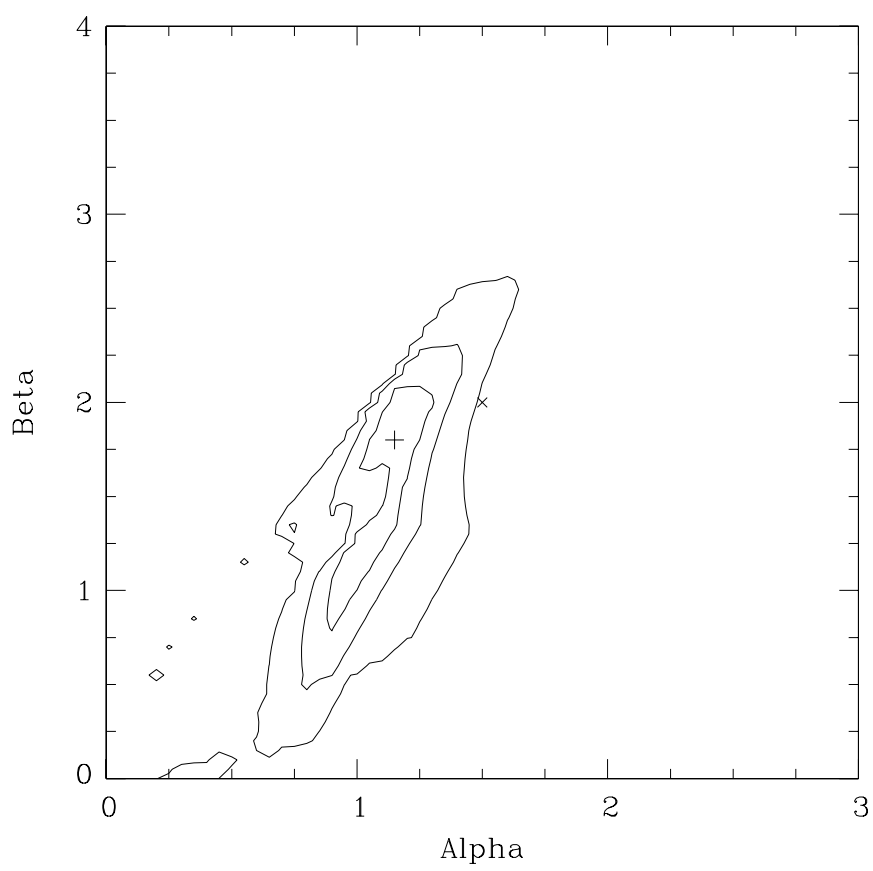

FIG. 7.- Same as Fig. 6, except that NGC 6397 is also excluded from the data. dence errors) indicates that most NSs in qLMXBs have not accreted $\sim 0.5 M_{\odot}$ from their companions. This is in general agreement with estimates of the masses of persistent LMXBs in several globular clusters $\left(1.8_{-0.4}^{+1.2} M_{\odot}\right.$; Grindlay et al. 1984 [recalculated in our $\S 3.1$ ]) and possible MSPs in 47 Tuc, thought to be their descendants $\left(1.26_{-0.19}^{+0.14} M_{\odot}\right.$; Grindlay et al. 2002 [recalculated in our $\S 3.1$ ]), both derived using the same method. It is also in agreement with the masses of pulsars (and MSPs in particular) derived by Thorsett \& Chakrabarty (1999), $1.35 \pm 0.04 M_{\odot}$, which indicates that very little mass is required to spin NSs up to millisecond periods. Although our finding does not rule out the possibility that some qLMXBs may have larger masses, it lends weight against the high-mass interpretation of the bright qLMXB X7 in 47 Tuc (Heinke et al. 2003c). As massive NSs should cool faster than less massive ones (e.g., Colpi et al. 2001), the brightest qLMXB (X7) should not be the most massive one.

Most of the qLMXBs in Table 2 are consistent with a $\sim 10 \mathrm{~km}$ NS radius when fitted with a hydrogen atmosphere model. A smaller radius might suggest that a polar cap was strongly heated, perhaps through ongoing accretion channeled by a magnetic field. A larger radius, as suggested for 47 Tuc X 7 and M30 A1, can be explained by a more massive $\left(>1.7 M_{\odot}\right)$ NS or by an alteration of opacity through continued accretion (see Heinke et al 2003c; P. M. Lugger et al. 2003, in preparation). Either possibility is of great interest. We note that our method in this paper of calculating the errors on the radius of these qLMXBs underestimates the true errors (see in 't Zand et al. 2001), and thus these radii should be taken only as a measure of consistency with expectations, not as rigorous constraints. See Heinke et al. (2003c), C. O. Heinke et al. (2003, in preparation), and P. M. Lugger et al. (2003, in preparation) for detailed constraints on the radius and/or mass of some of these qLMXBs.

The qLMXBs listed in Table 2 have very little or no power-law component, in contrast to field systems identified through their high-luminosity outbursts, which require $10 \%-40 \%$ of their $0.5-10 \mathrm{keV}$ emission in this component. Some field qLMXBs that have recently been accreting (KS 1731-260 [Wijnands et al. 2002b and references therein]; MXB 1659-298 [Wijnands et al. 2003]) allow a power-law component to constitute up to $\sim 25 \%$ of the $0.5-10 \mathrm{keV}$ flux, but they do not require it. The only globular cluster qLMXB to require this component, NGC $6440 \mathrm{CX} 1$, is the only qLMXB in our sample to have experienced a recorded outburst. This gives additional support to the suggestion by Heinke et al. (2003c) that the strength of the power-law component may be a measure of continuing low-level accretion. We note that field qLMXBs that have shown intrinsic variability on short timescales (Cen X-4 [Campana et al. 1997; Rutledge et al. 2003], Aq1 X-1 [Rutledge et al. 2002b]) indicative of continued accretion clearly show this power-law component. However, source 26 in M28 shows variability on a timescale of months without evidence of this power-law component (Becker et al. 2003), which does not fit this paradigm. However, this variability may be due to changing $N_{\mathrm{H}}$ column depth. No qLMXB without a power-law spectral component has yet been shown to require variability in its thermal component, suggesting that the thermal emission in these systems is entirely due to deep crustal heating (Brown et al. 1998). 


\subsection{Luminosity Information}

We use the theoretical scaling of qLMXB formation with central density and core radius (see $\S \S 3.3$ and 4.3) to calculate relative values of $\Gamma$ for the clusters in Table 1 as percentages of the total formation rate in the Galactic globular cluster system. We can extrapolate from the studied clusters to the remaining globular clusters in the catalog of Harris (1996) and thus estimate that roughly 95 accreting NS systems may be found among the entire Galactic globular cluster system, in agreement with the estimate of Pooley et al. (2003). As discussed in $\S 4.4$ below, unusual dynamical histories of some clusters may increase this number slightly. Thirty-eight accreting NS systems have now been identified (including the other 11 bright LMXBs and the qLMXB in NGC 6652). Seven times more qLMXBs in globular clusters are inferred than have been seen in outburst.

Wijnands et al. (2001, 2002a, 2003) suggest that there exists a population of qLMXBs with long-duration outbursts $(>10 \mathrm{yr})$ and extremely long dormant periods (thousands of years). This is required to explain the low quiescent flux from several qLMXBs that have been accreting for many years and thus would otherwise be expected to have very hot cores (Brown et al. 1998). Pfahl, Rappaport, \& Podsiadlowski (2003) suggest that irradiation-induced mass-transfer cycles, with long periods of dormancy, may be required to account for the apparent lack of LMXBs compared with the number of MSPs in the Galaxy. Our evidence that most accreting NSs in globular clusters are in deep quiescence supports the picture of long dormant periods suggested by Wijnands, Pfahl, and others, although globular cluster systems may be very different from field systems.

Chandra observations have been sufficiently sensitive to observe soft qLMXBs in many clusters below $\sim 1 \times 10^{32}$ ergs $\mathrm{s}^{-1}$ (all clusters in Table 1 except Terzan 5, M13, and probably NGC 6440), but they have not been seen. Assuming $10 \mathrm{~km}$ radii in the spectral fits leads us to conclude that no bolometric (redshifted) luminosities are below $2.3 \times 10^{32}$ ergs $s^{-1}$. This lower LF cutoff implies a lower limit to the time-averaged mass-transfer rate of these systems in the Brown et al. (1998) model. If enhanced neutrino cooling is not active and the mass transfer is mostly conservative, the bolometric luminosity range of qLMXBs given in Table 2 translates to time-averaged mass-transfer rates of $3 \times 10^{-12}$ to $7 \times 10^{-11} M_{\odot} \mathrm{yr}^{-1}$ (the latter for X7 in $47 \mathrm{Tuc}$ ). (We note that the revised distance to NGC 6397 calculated in Gratton et al. [2003] reduces the $0.5-2.5 \mathrm{keV}$ luminosity of its qLMXB U24 to $7 \times 10^{31}$ ergs s$^{-1}$ and thus blurs this cutoff.)

This range may represent the actual mass-transfer rates or may be an underestimate due to enhanced neutrino cooling (e.g., Colpi et al. 2001) or nonconservative mass transfer. We note that the larger number is a factor of a few less than the disk stability criterion for systems of periods similar to X5 (8 hr) in 47 Tuc (King 2003). Significantly larger mass-transfer rates would cause persistent emission instead of transient behavior. Taking these rates at face value, two explanations are plausible. Part of this range of masstransfer rates might be supplied by initially evolved secondaries, i.e., originally intermediate-mass X-ray binaries, as suggested to predominate by recent work (Pfahl et al. 2003). Alternatively, extremely old postminimum systems will naturally generate low mass-transfer rates below $10^{-11} M_{\odot} \mathrm{yr}^{-1}$ (King 2000). In this picture, the lower lumi- nosity limit is attributed to the finite age of the systems, less than a Hubble time (L. Bildsten 2003, private communication). We note that this luminosity range can be taken as support for the Brown et al. (1998) deep crustal heating model. An even lower thermal luminosity has been observed from the (low mass transfer, $\dot{m} \sim 5 \times 10^{-12} M_{\odot} \mathrm{yr}^{-1}$ ) millisecond X-ray pulsar SAX J1808 and has been taken to imply enhanced neutrino cooling from the core (Campana et al. 2002). If such enhanced cooling is common among globular cluster qLMXB systems, the time-averaged mass-transfer rates may be higher than we have calculated.

\subsection{Distribution among Globular Clusters}

Several methods of production of globular cluster binary $\mathrm{X}$-ray sources have been suggested. Normal evolution from primordial binaries is not a reasonable explanation for NS systems (Clark 1975) but may be able to explain some CVs and ABs (Verbunt \& Meylan 1988; Davies 1997). The numbers of such systems would depend on the initial mass of the cluster and the primordial binary fraction and would be suppressed by the destruction of wide binaries in close encounters (Davies 1997). Tidal capture of main-sequence stars by NSs or white dwarfs may generate large numbers of short-period systems (e.g., di Stefano \& Rappaport 1994), while exchange encounters will tend to inject NSs into longer period primordial binaries (e.g., Hut et al. 1991). Both of the latter mechanisms predict a rate of formation of NS binaries $\Gamma$ proportional to $\rho_{c}^{2} r_{c}^{3} / \sigma \propto \rho_{c}^{1.5} r_{c}^{2}$, where $\rho_{c}$ is the central luminosity density, $r_{c}$ is the core radius, and $\sigma$ the central velocity dispersion (Verbunt \& Hut 1987; Verbunt 2003). This assumes that the mass-to-light ratio is similar in the cores of different globular clusters and that most of the encounters happen within the core. The second assumption is a good approximation for King model clusters because of the steep density decline outside the core, but it is less accurate for core-collapsed clusters with a less steep decline outside the core. A more accurate calculation, as performed in Pooley et al. (2003), integrates the density distribution out to large radii, using the best surface profiles available. Our method does allow us, however, to investigate the dependence of X-ray source formation on core density and core size separately.

Our simple method does not account for increased NS density in the core due to mass segregation (Verbunt \& Meylan 1988) or for escape of NSs from globular cluster potential wells of different depth (see Pfahl et al. 2002). It also carries the potential for significant bias in that we analyze only a small sample of clusters, generally selected because of the existence of known X-ray sources and perhaps not representative of the general globular cluster system. With these caveats, we proceed.

Our test of the distribution of globular cluster NS systems in Figure 3 indeed shows compatibility with $\Gamma \propto \rho_{c}^{1.5} r_{c}^{2}$, as predicted by either tidal capture or exchange encounters. This result agrees with the results of Verbunt \& Hut (1987) on bright cluster LMXBs and the simpler tests of Gendre et al. (2003b) and Pooley et al. (2003) on qLMXBs in globular clusters. We note that this result is also compatible with the results of Johnston, Kulkarni, \& Phinney (1992) on recycled pulsars (the products of accreting NS systems) and those of Johnston \& Verbunt (1996) on globular cluster low-luminosity X-ray sources (the brightest of which are predominantly qLMXBs) when their neglect of the velocity 
dispersion $\sigma \propto \rho^{0.5} r_{c}$ is considered (as noted in Verbunt 2003). Grindlay (1996), using measured velocity dispersions, indeed showed correlation of dim source numbers with the theoretical scaling.

For the harder sources (predominantly CVs), we do not find agreement with the theoretically predicted formation rate from close encounters. Figures 4-6 show a weaker dependence on the central density for hard sources than for qLMXBs. The density dependence of the hard sources is better fitted by $\Gamma \propto \rho_{c}^{\alpha} r_{c}^{2}$ with $\alpha \sim 1.1-1.3$ than by $\alpha \sim 1.5$ as for qLMXBs. One suggestion for the lower density dependence is that high-density environments destroy CVs preferentially, possibly by encounters with NSs (e.g., Pooley et al. 2002b; Verbunt 2003), especially during the core collapse process. NGC 6397, the densest globular cluster yet studied, has an apparent excess of X-ray sources (Table 1; Pooley et al. 2003), which suggests the opposite conclusion. However, NGC 6397 may be unusual for other reasons (see $\S 4.4)$.

The formation of some CVs in globular clusters from primordial, undisturbed binaries (especially in massive, moderately dense clusters like $\omega$ Cen; Davies 1997; Verbunt 2003) may partially explain the weaker dependence on density. Many sources outside the half-mass radius of $\omega$ Cen may be CVs, two of them brighter than $L_{\mathrm{X}}=10^{32} \mathrm{ergs} \mathrm{s}^{-1}$ (Cool et al. 2002; Gendre et al. 2003a). Davies (1997) indeed predicts that CVs from primordial binaries should exist in the outer regions of this unrelaxed globular cluster. However, even if four bright primordial CVs exist in $\omega$ Cen, the masses of the other globular clusters in Table 1 are so much smaller that most of their bright CVs must not be primordial. According to Pryor \& Meylan (1993), $\omega$ Cen is as massive as all the other globular clusters with bright CVs (except Ter 5 and NGC 6440) put together. These other clusters have 21 bright CVs, so we infer that most of these $\mathrm{CVs}$ are not primordial.

\subsection{Other Clusters and Processes}

Our analysis of X-ray source distributions is consistent with X-ray imaging studies of some dense high- $\Gamma$ clusters (Liller 1 [Homer et al. 2001]; M15 [Hannikainen et al. 2003]) that suggest numerous $\mathrm{X}$-ray sources in the luminosity range of qLMXBs. However, several clusters with low predicted collision rates seem to have more X-ray sources than expected on the basis of their structural parameters; besides NGC 6397, these include NGC 6712, NGC 288, NGC 6652, and Terzan 1. Other dynamical processes may be at work in these clusters.

Analysis of the core-collapse process suggests that binaries in the core release energy to passing stars by " hardening" into tighter orbits, thus slowing the collapse process before their ultimate ejection or merger (Hut et al. 1992; Fregeau et al. 2003). The hardening of binaries during core collapse might be expected to generate increased masstransfer rates and thus X-ray emission. As core collapse proceeds, main-sequence binaries will be destroyed, but NSs should be preferentially exchanged into binaries. The numbers of each kind of X-ray binary existing at any one time may thus be a function of the cluster dynamical history, as well as current structure. Differences in dynamical history may explain the unusually large numbers of CVs (and NS systems?) in NGC 6397 (see Table 1) as compared with the similar core-collapsed globular clusters M30 and NGC
6544. The latter, while not yet surveyed by Chandra, has a ROSAT upper limit of $L_{\mathrm{X}}=5.9 \times 10^{31} \mathrm{ergs} \mathrm{s}^{-1}$ (Verbunt 2001) above which five sources exist in NGC 6397. NGC 6397 has recently been shown to be depleted in binaries compared with several other clusters (Cool \& Bolton 2002), while it appears to have an abundance of X-ray sources. NGC 6397 also seems to show an unusually flat CV LF, compared with 47 Tuc and other clusters in general $(\S 3.2$; Pooley et al. 2002b).

The cluster NGC 6712 (which contains a bright persistent ultracompact LMXB) shows evidence for multiple accreting binaries and blue stragglers despite its relatively low density and $\Gamma(\Gamma=0.13)$ (Ferraro et al. 2000; Paltrinieri et al. 2001). The path of NGC 6712's orbit through the Galactic bulge suggests that it experiences strong tidal stripping from the Galactic potential (Dauphole et al. 1996). NGC 6712's declining mass function below the turnoff (de Marchi et al. 1999; Andreuzzi et al. 2001) gives strong evidence that it has been stripped of $\geq 99 \%$ of its initial mass (Takahashi \& Portegies Zwart 2000). An analysis of the orbits and structure of 38 globular clusters by Dinescu, Girard, \& van Altena (1999) indicates that NGC 6712, NGC 6397, NGC 6121, NGC 288, Palomar 5, and possibly M80 have very high destruction rates. Ferraro et al. (2000) thus suggest that NGC 6712 is only the fossil remnant core of a once very massive cluster, heavily enriched in compact objects and binaries as a result of mass segregation. Disrupting globular clusters should be generally marked by an apparent excess of massive stars, binaries, and binary products (such as X-ray sources or blue stragglers), which will remain segregated in the core while the outer halo is stripped. This destruction process should deposit X-ray binaries from globular clusters into the Galactic bulge (Grindlay 1985).

NGC 6652 and Terzan 1 each possess a bright LMXB, although their collision rates are very low $(\Gamma=0.18$ and 0.008 , respectively). In addition, they are home to at least three and one additional X-ray sources above $\sim 5 \times 10^{32}$ ergs s${ }^{-1}$, respectively (Heinke et al. 2001; Wijnands et al. 2002a). The orbits of NGC 6652 and Terzan 1 have not yet been calculated, but Idiart et al. (2002) note that Terzan 1 seems to have captured metal-rich stars from the bulge, implying that it was once much more massive. Two of NGC 6652's X-ray sources, both probable NS systems, are well outside the core (Heinke et al. 2001). Although NGC 6652 does not show signs of core collapse, this implies an unusual dynamical state. Analysis of archival HST images to measure the stellar LF and surface profile of NGC 6652 could test this. NGC 6652 and Terzan 1 could be remnant cores of initially much more massive globular clusters on their way to destruction.

Several of the other clusters with high destruction rates show low-mass star depletion (NGC 6121 [Kanatas et al. 1995], NGC 288 [Bellazzini et al. 2002b], and NGC 6397 [Piotto, Cool, \& King 1997]). Palomar 5 shows both lowmass star depletion (Grillmair \& Smith 2001) and tidal tails (Odenkirchen et al. 2001). These facts suggest that cluster destruction processes may have concentrated these clusters' X-ray populations as well, although not as severely as NGC 6712. Such processes have recently been suggested by Pooley et al. (2003) to explain the apparent excess of X-ray sources in NGC 6397. Disentangling the effects of cluster destruction and core collapse on binary production will probably require significant theoretical work. 
The very loose cluster NGC 288 has an X-ray source with $L_{\mathrm{X}} \sim 3 \times 10^{32}$ from ROSAT HRI data (Verbunt 2001), even though it is very poor, with $\Gamma=0.005$. NGC 288 shows centrally concentrated binary stars and blue stragglers (Bellazzini et al. 2002a; Ferraro et al. 2003). However, Bellazzini et al. (2002a) think that it would be very difficult to reexpand NGC 288 to its current low density after compressing the core sufficiently to produce collisional products. They and Ferraro et al. (2003) instead suggest that NGC 288's large number of blue stragglers is the result of primordial binary evolution, like the halo blue stragglers in M3 (Ferraro et al. 1997), and may be due to an initially high binary fraction. The high destruction rate of NGC 288 suggests that its binary fraction may be enhanced by the removal of lower mass single stars from the halo during its repeated disk shocks. It will be of great interest to see whether the same processes that generate large numbers of blue stragglers in NGC 288 also produce numerous X-ray sources detectable in recent Chandra observations (PI: W. H. G. Lewin).

Metallicity has been suggested to have an effect on the luminosity of extragalactic globular cluster X-ray sources (e.g., Kundu, Maccarone, \& Zepf 2002). This metallicity effect may be due to differences in cluster initial mass functions (Grindlay 1993) or to the larger radii of metal-rich stars increasing both tidal capture and Roche lobe overflow rates (Bellazzini et al. 1995). More massive clusters should be better able to retain NSs after their formation kicks (Pfahl et al. 2002), which will affect the numbers of MSPs as well as qLMXBs. Neither mass nor metallicity has obvious effects on the Galactic globular clusters studied here. Analyzing the effects of all the above factors on the various globular cluster populations will require deep observations of a number of clusters with very different parameters, paired with deep radio and optical data sets to clearly identify different source types below the luminosities discussed here.

\section{CONCLUSIONS}

We have created a catalog of known qLMXBs containing NSs in globular clusters, adding three probable qLMXBs in NGC 6440 to those already known. We have reanalyzed those qLMXBs in archived Chandra globular cluster observations using the hydrogen atmosphere models of Lloyd (2003) and find general consistency with $10 \mathrm{~km}$ radii. The hard power-law component required in the spectra of many field qLMXBs is absent in most globular cluster qLMXBs, with the notable exception of the recently active transient in NGC 6440. The radial distribution of these qLMXBs within their globular clusters is consistent with a mass of $1.5_{-02}^{+0.3}$ $M_{\odot}$. This is as expected for accreting NS systems and suggests that the NSs do not grow significantly in mass. Globular cluster qLMXBs range in luminosity from $\sim 10^{32}$ ergs s ${ }^{-1}$ up to a few $10^{33} \mathrm{ergs} \mathrm{s}^{-1}$. Quiescent LMXBs below $10^{32} \mathrm{ergs} \mathrm{s}^{-1}$ would have been identifiable in most clusters, so the cutoff implies a lower limit to the time-averaged mass accretion rate. This range of luminosities is consistent with the Brown et al. (1998) model for qLMXB emission, as higher mass-transfer rates would lead to persistent systems and significantly lower rates would probably require systems older than a Hubble time.

The luminosity function of globular cluster qLMXBs is consistent with the LF of globular cluster sources analyzed by Johnston \& Verbunt (1996), which is not surprising since they are usually the brightest sources in a cluster. The LFs of harder sources above $10^{31} \mathrm{ergs}^{-1}$, which are mostly CVs, appear to vary between clusters, suggesting an influence of metallicity or core collapse on CV properties. The numbers of qLMXBs in different clusters are consistent with the relative numbers of close encounters, allowing either tidal capture or exchange encounters as a mode of production. This suggests that the total number of accreting NSs in globular clusters is near 100. The harder sources, however, show a lesser dependence on density, suggesting that dense environments may tend to destroy CVs. The core collapse process could be responsible for differences in the numbers and types of X-ray binaries between NGC 6397 and similar clusters. Tidal destruction or evaporation of clusters may leave substantial numbers of X-ray sources in apparently poor clusters.

This study has begun to probe individual source populations across different globular clusters and test the effects of varying central density and core radius on the properties of two populations, qLMXBs and CVs. Future Chandra observations will allow us to test the effects of metallicity, cluster mass, and other dynamical processes in clusters. Deep optical and radio data sets are also allowing identification and study of populations of ABs and MSPs. In addition to understanding the properties of these binaries, this work offers an opportunity for a deeper understanding of globular cluster evolution.

We are very grateful to D. Pooley for communicating results of several globular cluster studies to us before publication. We also thank L. Bildsten for useful discussions, A. Kong and D. Pooley for comments on the manuscript, and the anonymous referee for useful suggestions. C. O. H. acknowledges support from Chandra grant GO2-3059A.

\section{REFERENCES}

Anderson, A. J. 1997, Ph.D. thesis, Univ. California, Berkeley

Andreuzzi, G., De Marchi, G., Ferraro, F. R., Paresce, F., Pulone, L., \& Buonanno, R. 2001, A\&A, 372, 851

Anthony-Twarog, B. J., \& Twarog, B. A. 2000, AJ, 120, 3111

Arnaud, K. A. 1996, in ASP Conf. Ser. 101, Astronomical Data Analysis Software and Systems V., ed. G. Jacoby \& J. Barnes (San Fancisco: ASP), 17

Balucinska-Church, M., \& McCammon, D. 1992, ApJ, 400, 699

Becker, W., et al. 2003, ApJ, 594, 798

Bellazzini, M., Fusi Pecci, F., Messineo, M., Monaco, L., \& Rood, R. T. 2002a, AJ, 123, 1509

. 2002b, AJ, 123, 2541

Bellazzini, M., Pasquali, A., Federici, L., Ferraro, F. R., \& Fusi Pecci, F. 1995, ApJ, 439, 687
Brown, E. F., Bildsten, L., \& Rutledge, R. E. 1998, ApJ, 504, L95

Campana, S., Colpi, M., Mereghetti, S., Stella, L., \& Tavani, M. 1998, A\&A Rev., 8, 279

Campana, S., Mereghetti, S., Stella, L., \& Colpi, M. 1997, A\&A, 324, 941

Campana, S., et al. 2002, ApJ, 575, L15

Cardelli, J. A., Clayton, G. C., \& Mathis, J. S. 1989, ApJ, 345, 245

Clark, G. W. 1975, ApJ, 199, L143

Cohn, H. N., Lugger, P. M., Grindlay, J. E., \& Edmonds, P. D. 2002, ApJ, 571,818

Colpi, M., Geppert, U., Page, D. \& Possenti, A. 2001, ApJ, 548, L175

Cool, A. M., \& Bolton, A. S. 2002, in ASP Conf. Ser. 263, Stellar Collisions, Mergers, and Their Consequences, ed. M. M. Shara (San Francisco: ASP), 163 
Cool, A. M., Grindlay, J. E., Cohn, H. N., Lugger, P. M., \& Slavin, S. D. 1995, ApJ, 439, 695

Cool, A. M., Haggard, D. Carlin, J. L. 2002, in ASP Conf. Ser. 265 , $\omega$ Centauri : A Unique Window into Astrophysics, ed. F. van Leeuwen, J. D. Hughes, \& G. Piotto, (San Francisco: ASP), 277

D’Amico, N., Possenti, A., Fici, L., Manchester, R. N., Lyne, A. G., Camilo, F., \& Sarkissian, J. 2002, ApJ, 570, L89

Dauphole, B., Geffert, M., Colin, J., Ducourant, C., Odenkirchen, M., \& Tucholke, H.-J. 1996, A\&A, 313, 119

Davies, M. B. 1997, MNRAS, 288, 117

de Marchi, G., Leibundgut, B., Paresce, F., \& Pulone, L. 1999, A\&A, 343, L9

Dinescu, D. I., Girard, T. M., \& van Altena, W. F. 1999, AJ, 117, 1792

Di Stefano, R., \& Rappaport, S. 1994, ApJ, 423, 274

Djorgovski, S. 1993, in ASP Conf. Ser. 50, Structure and Dynamics of Globular Clusters, ed. S, G, Djorgovski \& G. Meylan (San Francisco: ASP), 373

Edmonds, P. D., Gilliland, R. E., Camilo, F., Heinke, C. O., \& Grindlay, J. E. 2002a, ApJ, 579, 741

Edmonds, P. D., Gilliland, R. E., Heinke, C. O., \& Grindlay, J. E. 2003a, ApJ, 596, 1177

.2003b, ApJ, 596, 1197

Edmonds, P. D., Gilliland, R. L., Heinke, C. O., Grindlay, J. E., \& Camilo, F. 2001, ApJ, 557, L57

Edmonds, P. D., Heinke, C. O., Grindlay, J. E., \& Gilliland, R. L. 2002b, ApJ, 564, L17

Ferraro, F. R., Paltrinieri, B., Paresce, F., \& De Marchi, G. 2000, ApJ, 542, L29

Ferraro, F. R., Possenti, A., D’Amico, N., \& Sabbi, E. 2001, ApJ, 561, L93

Ferraro, F. R., Sills, A., Rood, R. T., Paltrinieri, B., \& Buonanno, R. 2003, ApJ, 588, 464

Ferraro, F. R., et al. 1997, A\&A, 324, 915

Fregeau, J. M., Gürkan, M. A., Joshi, K. J., \& Rasio, F. A. 2003, ApJ, 593, 772

Gehrels, N. 1986, ApJ, 303, 336

Gendre, B., Barret, D., \& Webb, N. A. 2003a, A\&A, 400, 521 2003b, A\&A, 403, 11

Giacconi, R., et al. 2001, ApJ, 551, 624

Gratton, R., Bragaglia, A., Carretta, E., Clementini, G., Desidera, S., Grundahl, F., \& Lucatello, S. 2003, A\&A, 408, 529

Grillmair, C. J., \& Smith, G. H. 2001, AJ, 122, 3231

Grindlay, J. E. 1993, in ASP Conf. Ser. 48, The Globular Cluster-Galaxy Connection, ed. G. H. Smith \& J. P. Brodie (San Francisco: ASP), 156 . 1996, in IAU Symp. 174, Dynamical Evolution of Star Clusters, ed. P. Hut \& J. Makino (Dordrecht: Kluwer), 171

1985, in IAU Symp. 113, Dynamics of Star Clusters, ed. J. Goodman \& P. Hut (Dordrecht: Kluwer). 43

Grindlay, J. E., Camilo, F., Heinke, C. O., Edmonds, P. D., Cohn, H., \& Lugger, P. 2002, ApJ, 581, 470

Grindlay, J. E., Cool, A. M., Callanan, P. J., Bailyn, C. D., Cohn, H. N., \& Lugger, P. M. 1995, ApJ, 455, L47

Grindlay, J. E., Heinke, C. O., Edmonds, P. D., \& Murray, S. S. 2001a, Science, 292, 2290 (GHE01a)

Grindlay, J. E., Heinke, C. O., Edmonds, P. D., Murray, S. S., \& Cool, A. M. 2001b, ApJ, 563, L53 (GHE01b)

Grindlay, J. E., Hertz, P., Steiner, J. E., Murray, S. S., \& Lightman, A. P. 1984, ApJ, 282, L13

Hannikainen, D. C., Charles, P. A., van Zyl, L., \& Kong, A. K. H. 2003, MNRAS, submitted

Harris, W. E. 1996, AJ, 112, 1487

Heinke, C. O., Edmonds, P. D., \& Grindlay, J. E. 2001, ApJ, 562, 363

Heinke, C. O., Edmonds, P. D., Grindlay, J. E., Lloyd, D. A., Cohn, H. N., \& Lugger, P. M. 2003a, ApJ, 590, 809

Heinke, C. O., Grindlay, J. E., Edmonds, P. D., Lloyd, D. A., Murray, S. S., Cohn, H. N., \& Lugger, P. M. 2003b, ApJ, 590, 809

Heinke, C. O., Grindlay, J. E., Lloyd, D. A., \& Edmonds, P. D. 2003c, ApJ, 588,452
Hertz, P., \& Grindlay, J. E. 1983, ApJ, 275, 105

Homer, L., Deutsch, E. W., Anderson, S. F., \& Margon, B. 2001, AJ, 122, 2627

Hut, P., Murphy, B. W.. \& Verbunt, F. 1991, A\&A, 241, 137

Hut, P., et al. 1992, PASP, 104, 981

Idiart, T. P., Barbuy, B., Perrin, M.-N., Ortolani, S., Bica, E., \& Renzini, A. 2002, A\&A, 381, 472

in 't Zand, J. J. M., van Kerkwijk, M. H., Pooley, D., Verbunt, F., Wijnands, R., \& Lewin, W. H. G. 2001, ApJ, 563, L41

in 't Zand, J. J. M., et al. 1999, A\&A, 345, 100

Johnston, H. M., Kulkarni, S. R., \& Phinney, E. S. 1992, in X-Ray Binaries and Recycled Pulsars, ed. E. P. J. van den Heuvel \& S. A. Rappaport (Dordrecht: Kluwer), 349

Johnston, H. M., \& Verbunt, F. 1996, A\&A, 312, 80

Kanatas, I., Griffiths, W. K., Dickens, R. J., \& Penny, A. J. 1995, MNRAS, 272,265

Katz, J. I. 1975, Nature, 253, 698

King, A. R. 2000, MNRAS, 315, L33

2003, preprint (astro-ph/0301118)

King, I. R. 1966, AJ, 71, 64

King, I. R., Anderson, J., Cool, A. M., \& Piotto, G. 1998, ApJ, 492, L37

Kundu, A., Maccarone, T. J., \& Zepf, S. E. 2002, ApJ, 574, L5

Liedahl, D. A., Osterheld, A. L., \& Goldstein, W. H. 1995, ApJ, 438, L115

Lloyd, D. A. 2003, MNRAS, submitted

Lugger, P. M., Cohn, H. N., \& Grindlay, J. E. 1995, ApJ, 439, 191

Nowak, M. A., Heinz, S., \& Begelman, M. C. 2002, ApJ, 573, 778

Odenkirchen, M., et al. 2001, ApJ, 548, L165

Paltrinieri, B., Ferraro, F. R., Paresce, F., \& De Marchi, G. 2001, AJ, 121, 3114

Pfahl, E., Rappaport, S., \& Podsiadlowski, P. 2002, ApJ, 573, 283 2003, ApJ, 597, 1036

Piotto, G., Cool, A. M., \& King, I. R. 1997, AJ, 113, 1345

Pooley, D., et al. 2002a, ApJ, 569, 405

2002b, ApJ, 573, 184 2003, ApJ, 591, L131

Predehl, P., \& Schmitt, J. H. M. M. 1995, A\&A, 293, 889

Pryor, C., \& Meylan, G. 1993, in ASP Conf. Ser. 50, Structure and Dynamics of Globular Clusters, ed. S. G. Djorgovski \& G. Meylan (San Francisco: ASP), 357

Rutledge, R. E., Bildsten, L., Brown, E. F., Pavlov, G. G., \& Zavlin, V. E. 2001, ApJ, 551, 921

.2002a, ApJ, 578, 405 2002 b, ApJ, 577, 346

2003, AAS/HEAD Meeting, 35, 42.03

Sosin, C. A. 1997, Ph.D. thesis, Univ. California, Berkeley

Takahashi, K., \& Portegies Zwart, S. F. 2000, ApJ, 535, 759

Thorsett, S. E., \& Chakrabarty, D. 1999, ApJ, 512, 288

van Leeuwen, F., \& Le Poole, R. S. 2002, in ASP Conf. Ser. 265, $\omega$ Centauri: A Unique Window in Astrophysics, ed. F. van Leeuwen,

J. D. Hughes \&, G. Piotto (San Francisco: ASP), 41

Verbunt, F. 2001, A\&A, 368, 137

-. 2003 in ASP Conf. Ser. 296, New Horizons in Globular Cluster Astronomy, ed. G. Piotto, G. Meylan, S. G. Djorgovski, \& M. Riello (San Francisco: ASP), in press (astro-ph/0210057)

Verbunt, F., Elson, R., \& van Paradijs, J. 1984, MNRAS, 210, 899

Verbunt, F., \& Hut, P. 1987, in IAU Symp. 125, Origin and Evolution of Neutron Stars, ed. D. J. Helfand \& J.-H. Huang (Dordrecht: Reidel), 187 Verbunt, F., \& Meylan, G. 1988, A\&A, 203, 297

Webb, N. A., Gendre, B., \& Barret, D. 2002, A\&A, 381, 481

Wijnands, R., Guainazzi, M., van der Klis, M., \& Méndez, M. 2002b, ApJ, 573, L45

Wijnands, R., Heinke, C. O., \& Grindlay, J. E. 2002a, ApJ, 572, 1002

Wijnands, R., Miller, J. M., Markwardt, C., Lewin, W. H. G., \& van der Klis, M. 2001, ApJ, 560, L159

Wijnands, R., Nowak, M. Miller, J. M., Homan, J., Wachter, S., \& Lewin, W. H. G. 2003, ApJ, 594, 952

Zavlin, V. E., Pavlov, G. G., \& Shibanov, Yu. A. 1996, A\&A, 315, 141 